\title{
Protective antibodies against Eastern equine encephalitis virus bind to epitopes in domains $A$ and $B$ of the E2 glycoprotein
}

\author{
Arthur S. Kim 1,2, S. Kyle Austin1, Christina L. Gardner ${ }^{3,9}$, Adam Zuiani², Douglas S. Reed ${ }^{3}$, \\ Derek W. Trobaugh ${ }^{3}$, Chengqun Sun ${ }^{3}$, Katherine Basore ${ }^{2}$, Lauren E. Williamson ${ }^{4}$, James E. Crowe Jr. ${ }^{4}$, \\ Mark K.Slifka ${ }^{5}$, Daved H. Fremont $\mathbb{B}^{2,6,7}$, William B. Klimstra ${ }^{3}$ and Michael S. Diamond $\mathbb{B}^{1,2,7,8 \star}$
}

\begin{abstract}
Eastern equine encephalitis virus (EEEV) is a mosquito-transmitted alphavirus with a high case mortality rate in humans. EEEV is a biodefence concern because of its potential for aerosol spread and the lack of existing countermeasures. Here, we identify a panel of 18 neutralizing murine monoclonal antibodies (mAbs) against the EEEV E2 glycoprotein, several of which have 'elite' activity with 50 and $99 \%$ effective inhibitory concentrations $\left(\mathrm{EC}_{50}\right.$ and $\left.\mathrm{EC}_{99}\right)$ of less than 10 and $100 \mathrm{ng} \mathrm{ml}^{-1}$, respectively. Alanine-scanning mutagenesis and neutralization escape mapping analysis revealed epitopes for these $m A b s$ in domains $A$ or $B$ of the E2 glycoprotein. A majority of the neutralizing mAbs blocked infection at a post-attachment stage, with several inhibiting viral membrane fusion. Administration of one dose of anti-EEEV mAb protected mice from lethal subcutaneous or aerosol challenge. These experiments define the mechanistic basis for neutralization by protective anti-EEEV mAbs and suggest a path forward for treatment and vaccine design.
\end{abstract}

\section{T} he EEEV is a mosquito-transmitted New World alphavirus in the Togaviridae family and is closely related to the Western (WEEV) and Venezuelan (VEEV) equine encephalitis viruses. Although relatively few human infections are reported annually, EEEV is one of the most severe mosquito-transmitted diseases with a $50-70 \%$ mortality rate and significant brain damage in most survivors $^{1-6}$. Florida is now considered one of the major sources of EEEV epidemics in the USA, with transmission occuring throughout the year ${ }^{7}$.

EEEV is an enveloped virus with a 11.5 kilobase singlestranded, positive-sense RNA genome that generates two RNA transcripts: a full-length genomic RNA; and a subgenomic RNA encoding the structural genes, C-E3-E2-6K-E1 ${ }^{8}$. After translation, the structural polypeptide C-E3-E2-6K-E1 is cleaved at the endoplasmic reticulum into the capsid protein and E3-E2-6K-E1. Additional protein processing in the endoplasmic reticulum and the Golgi apparatus results in transport of E2-E1 heterodimers to the plasma membrane ${ }^{9}$ where encapsidation of the genomic viral RNA occurs. The surface of the mature virion displays 80 spikes of trimers of E2-E1 heterodimers ${ }^{10}$. Structural studies of related alphaviruses have established an architecture with $T=4$ icosahedral symmetry ${ }^{10-12}$. The E2 glycoprotein projects from the viral surface and consists of three domains: $\mathrm{A}, \mathrm{B}$ and $\mathrm{C}^{11,12}$. Binding of EEEV E2 to poorly characterized host receptors is believed to initiate entry and endocytosis ${ }^{13}$. The acidic environment of the endosome induces conformational changes in the alphavirus E1 and E2 glycoproteins, which allow for the exposure of the fusion loop, insertion into the host membrane $\mathrm{e}^{11}$ and nucleocapsid escape into the cytoplasm.

Few anti-EEEV mAbs have been described ${ }^{14-16}$ and only one has protective activity in mice ${ }^{17}$. These anti-EEEV mAbs have been mapped using peptides to three linear epitopes on E2: the $\mathrm{N}$ terminus of domain $\mathrm{A}$; the $\mathrm{N}$ - and $\mathrm{C}$-terminal arches of domain $\mathrm{B}$; and the $\mathrm{C}$ terminus of domain $\mathrm{C}^{14,15}$. In comparison, the epitopes of several murine and human mAbs against VEEV, WEEV or the more distantly related arthritogenic alphaviruses, for example, chikungunya virus (CHIKV), with therapeutic efficacy in vivo have been mapped ${ }^{8,14,15,18,19}$. These neutralizing $\mathrm{mAbs}$ predominantly recognize epitopes in domains A (residues 58-80) or B (residues 180-215) of the E2 glycoprotein, and inhibit infection at multiple steps including viral attachment, entry, fusion and egress ${ }^{18-23}$.

We isolated and purified a panel of murine mAbs against EEEV. Among these, 18 type-specific mAbs neutralized EEEV infection with $50 \%$ effective inhibitory concentration $\left(\mathrm{EC}_{50}\right)$ values $<100 \mathrm{ng} \mathrm{ml}^{-1}$ and did not bind to WEEV or VEEV. Ten of these mAbs potently inhibited infection with $\mathrm{EC}_{50}$ values $<10 \mathrm{ng} \mathrm{ml}^{-1}$. In cell culture, most inhibited EEEV predominantly by blocking viral infection at a post-attachment step. We localized the epitopes of the majority of potently neutralizing mAbs to two solvent-exposed regions in domains $\mathrm{A}$ and $\mathrm{B}$ of the $\mathrm{E} 2$ glycoprotein. In vivo studies demonstrated that many of the neutralizing mAbs could protect mice against lethal subcutaneous or aerosol challenges by EEEV. Our results define the molecular basis for EEEV neutralization by protective mAbs and provide insight into the epitopes that could be

'Department of Medicine, Washington University School of Medicine, St. Louis, MO, USA. ${ }^{2}$ Pathology and Immunology, Washington University School of Medicine, St. Louis, MO, USA. ${ }^{3}$ Center for Vaccine Research, Department of Immunology, University of Pittsburgh, Pittsburgh, PA, USA. ${ }^{4}$ Vanderbilt Vaccine Center, Department of Pediatrics, and Department of Pathology, Microbiology, and Immunology, Vanderbilt University Medical Center, Nashville, TN, USA. ${ }^{5}$ Division of Neuroscience, Oregon National Primate Research Center, Oregon Health \& Science University, Beaverton, OR, USA. ${ }^{6}$ Biochemistry and Molecular Biophysics, Washington University School of Medicine, St. Louis, MO, USA. ${ }^{7}$ Molecular Microbiology, Washington University School of Medicine, St. Louis, MO, USA. ${ }^{8}$ The Andrew M. and Jane M. Bursky Center for Human Immunology and Immunotherapy Programs, Washington University School of Medicine, St. Louis, MO, USA. ${ }^{9}$ Present address: United States Army Research Institute for Infectious Diseases, Fort Detrick, MD, USA. *e-mail: diamond@wusm.wustl.edu 
targeted for immunotherapy and vaccine development against this highly lethal virus.

\section{Results}

Generation of anti-EEEV mAbs. We hypothesized that antibodies generated in the context of a live EEEV infection might have inhibitory activity. As EEEV is a biosafety level 3 select agent pathogen, performing B cell-myeloma cell fusions from infected animals presents technical challenges. To circumvent these issues, we engineered a chimeric biosafety level 2 pathogen that incorporates the non-structural genes and RNA replication control elements of a Sindbis virus (SINV, strain TR339) with the structural genes (C-E3E2-6K-E1) of an EEEV isolate (strain FL93-939) (Supplementary Fig. 1a ${ }^{24}$. SINV-EEEV replicated efficiently in cell culture but did not cause disease in outbred and $\mathrm{Irf3}^{-/}$immunodeficient inbred mice (Supplementary Fig. 1b-e, and see text that follows).

To enhance the replication and immunogenicity of the attenuated SINV-EEEV in vivo, we inoculated $\mathrm{Irf3}^{-1-} \mathrm{C} 57 \mathrm{BL} / 6 \mathrm{mice}^{25}$. After infection and homologous boosting four weeks later, serum from $\mathrm{Irf3}^{-1-}$ mice had robust neutralizing activity against SINVEEEV (end point titre $>1: 10,000$ ). Splenocytes were collected from mice, fused to myeloma cells and 76 hybridomas producing antiEEEV antibodies were isolated (Fig. 1a and Supplementary Table 1). Supernatant from 32 of the 76 hybridomas bound to EEEV virions purified from SINV-EEEV-infected cells and in a single end point dilution test, inhibited SINV-EEEV infection by $80 \%$ or more (Supplementary Table 1). These $32 \mathrm{mAbs}$ were isotyped (all of the immunoglobulin IgG2c or IgG3 subclass) and purified by protein A affinity chromatography for subsequent study.

We evaluated the purified mAbs for their ability to recognize the EEEV E2 glycoprotein. To do this, we purified recombinant EEEV E2 glycoprotein after expression in bacteria and oxidative refolding (Fig. 1b). Notably, 18 of $32 \mathrm{mAbs}$ bound to the recombinant E2 glycoprotein in an enzyme-linked immunosorbent assay (ELISA) (Fig. 1c). We also tested a set of $24 \mathrm{mAbs}$ for cross-reactivity with related VEEV or WEEV (55 and 56\% amino acid identity in the structural proteins). None of these anti-EEEV mAbs cross-reacted with the WEEV or VEEV structural proteins (Fig. 1d).

Neutralizing activity of $\mathbf{m A b s}$. To assess the inhibitory activity of the anti-EEEV mAbs more quantitatively, we performed focus reduction neutralization tests with Vero cells while maintaining $\mathrm{mAbs}$ in the medium both before and after virus inoculation (pre/ post-attachment, Fig. 2a,c). We determined the concentration of $\mathrm{mAb}$ that reduced infection by 50,90 or $99 \%\left(\mathrm{EC}_{50}, \mathrm{EC}_{90}\right.$ or $\mathrm{EC}_{99}$, see Table 1). Of the $33 \mathrm{mAbs}$ tested, 18 inhibited SINV-EEEV with $\mathrm{EC}_{50}$ values $<100 \mathrm{ng} \mathrm{ml}^{-1}$, and $10 \mathrm{mAbs}$ showed exceptional potency with $\mathrm{EC}_{50}$ values $<10 \mathrm{ng} \mathrm{ml}^{-1}$ and $\mathrm{EC}_{90}$ values $<100 \mathrm{ng} \mathrm{ml}^{-1}$. Four of these mAbs (EEEV-18, EEEV-69, EEEV-82, EEEV-86) had 'elite' neutralizing activity with $\mathrm{EC}_{99}$ values $<100 \mathrm{ng} \mathrm{ml}^{-1}$.

Antibody neutralization of alphaviruses can occur by inhibiting attachment, internalization or fusion, or by blocking assembly and budding ${ }^{18}$. To begin to define how the 11 most strongly neutralizing $\mathrm{mAbs}$ inhibited infection, we initially assessed whether they blocked virus attachment. Virus-mAb complexes were incubated with Vero cells at $4^{\circ} \mathrm{C}$; after extensive washing, viral RNA adsorbed to cells was detected by quantitative reverse-transcription $\mathrm{PCR}^{18,26}$. Notably, the four anti-EEEV mAbs with 'elite' neutralizing activity (EEEV-18, EEEV-69, EEEV-82, EEEV-86) did not reduce virus attachment (Fig. 2e). A modest (43-48\%) inhibition of attachment was observed for neutralizing mAbs EEEV-3 or EEEV-66, although statistical significance was not attained. As a positive control, preincubation of SINV-EEEV with soluble heparin, whose cell surface analogue heparan sulfate is an attachment factor for $\mathrm{EEEV}^{27}$, diminished virus binding to target cells in a dose-dependent manner (Fig. 2f). Incubation with higher concentrations of mAbs also failed to reduce virus attachment (Supplementary Fig. 2). We next performed post-attachment neutralization assays in which mAbs were incubated with SINV-EEEV after absorption to cells (postattachment, Fig. 2b,d). All of the potently neutralizing mAbs inhibited SINV-EEEV infection when added after the virus was bound to cells, suggesting that at least part of their inhibitory activity was at a post-attachment step. We next tested whether our neutralizing mAbs could inhibit viral fusion using a plasma membrane fusion-from-without (FFWO) assay ${ }^{28}$. After allowing viral attachment to Vero cells at $4^{\circ} \mathrm{C}, \mathrm{mAbs}$ were added and plasma membrane fusion was induced by a $37^{\circ} \mathrm{C}$ pulse in an acidic ( $\mathrm{pH}$ 5.5) medium. Subsequently, cells were propagated in medium supplemented with $20 \mathrm{mM} \mathrm{NH}_{4} \mathrm{Cl}$ to prevent de novo infection via the endocytic pathway, and were then stained for E2 antigen expression. Five of the mAbs tested (EEEV-3, EEEV-10, EEEV-18, EEEV-22 and EEEV-58) blocked virus plasma membrane fusion (Fig. $2 \mathrm{~g}$,h). For reasons that remain unclear (see Discussion), EEEV-66, EEEV-82, EEEV-102 and EEEV-107 paradoxically enhanced plasma membrane fusion of the virus.

Epitope mapping by alanine-scanning mutagenesis. We used alanine-scanning mutagenesis coupled with HEK-293T cell-based expression and flow cytometry ${ }^{1,29}$ to identify residues in the E2 glycoprotein required for $\mathrm{mAb}$ binding (Fig. 3a). Cells were transfected with plasmids encoding individual alanine (or serine for alanine residues) substitutions (360 residues) in the E2 gene in the context of a pE2-6K-E1 expression plasmid. We defined criti$\mathrm{cal}$ residues as those with $<25 \%$ binding to a given individual $\mathrm{mAb}$ that retained $>70 \%$ binding to an anti-EEEV oligoclonal antibody control (Table 1, Supplementary Fig. 3 and Supplementary Table 2). We excluded from analysis mutations of cysteine residues and substitutions that globally altered E2 conformation, as defined by reduced binding of an oligoclonal antibody. A majority (13 of 16) of the neutralizing mAbs tested mapped to the 'wing insertion' of domain A (residues 52-82) or the distal region of domain B $(\beta \text {-strands } A, B \text { and } E)^{11}$ of the E2 glycoprotein (Fig. 3a-c). The key loss-of-binding residues were highly conserved between the four (I, II, III and IV) EEEV subtypes (Fig. 3a). Although the domain $\mathrm{B}$ residues (I180, H181, S182, H213 and T215) required for $\mathrm{mAb}$ binding showed clear loss-of-binding phenotypes (Fig. 3d), some of the domain A residue changes (for example, D58, G59, D61 and M68) resulted in only partial loss-of-binding phenotypes (Fig. 3e). To extend these findings, we substituted selected residues in the A and $\mathrm{B}$ domains with bulkier and charged amino acids that might disrupt $\mathrm{mAb}$ interactions to a greater extent. We observed more profound loss-of-binding phenotypes when key domain $\mathrm{B}$ residues were substituted with arginine (Fig. 3f). Similarly, when the residues in domain A (D58, G59, D61, M68, K74 and L81) were mutated to arginine or glutamic acid, more pronounced loss of $\mathrm{mAb}$ binding phenotypes was observed with EEEV-5, EEEV-58, EEEV-66, EEEV-82, EEEV-102 and EEEV-107 (Fig. 3g, Supplementary Fig. 4 and Supplementary Table 3). Mapping of the domain A and B residues onto the CHIKV E3-E2-E1 glycoprotein complex structure revealed continuous solvent-exposed patches in each domain (Fig. 3b,c).

Epitope mapping by neutralization escape. Alanine-scanning mutagenesis failed to map the epitopes of three inhibitory mAbs (EEEV-18, EEEV-82 and EEEV-102). As an alternative approach, we selected for neutralization escape mutants. We passaged SINVEEEV in the presence of individual neutralizing mAbs until cytopathogenic effects were observed (3-4 passages), at which point the virus became resistant to neutralization. Remarkably, all three viral escape variants were reciprocally resistant to neutralization by the other mAbs in this group, suggesting they bound to an overlapping or shared epitope (Fig. 4a). To identify the escape muta- 


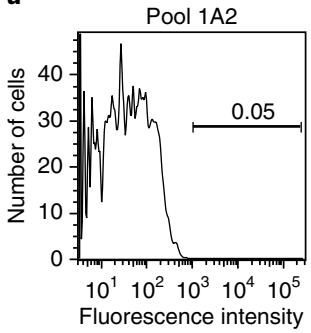

b
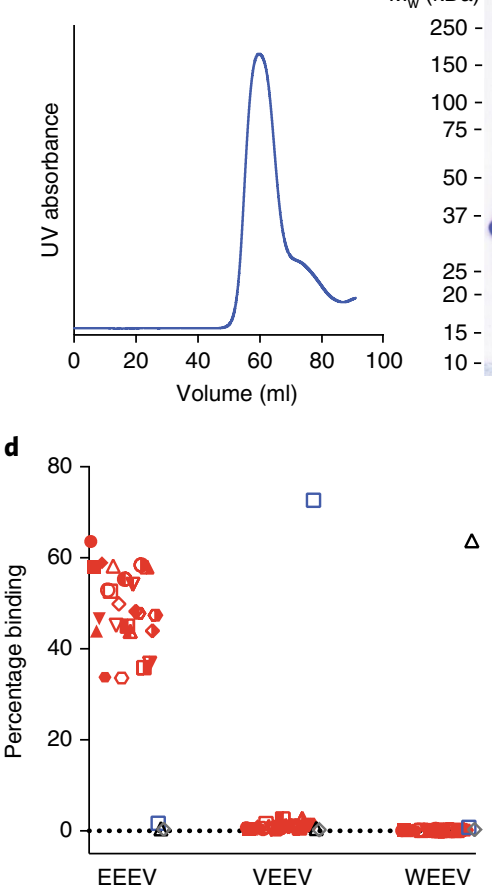

$M_{\mathrm{w}}(\mathrm{kDa})$
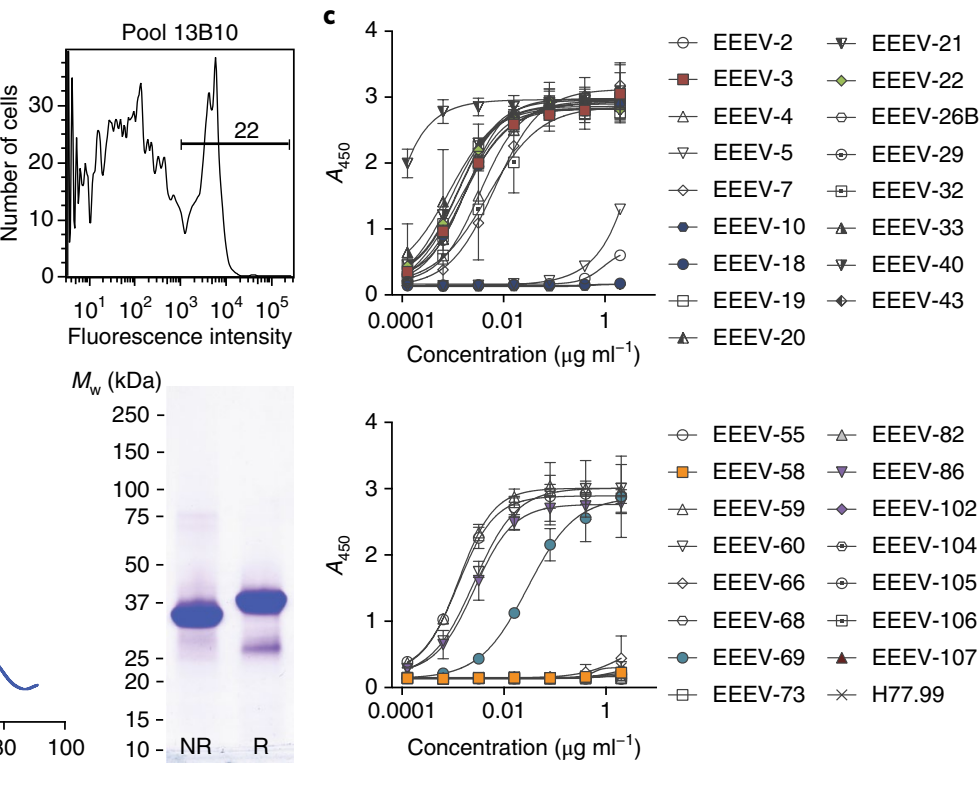

- - EEEV-55 $\triangle-$ EEEV-82

$\rightarrow$ EEEV-58 $\rightarrow$ EEEV-86

$\triangle$ EEEV-59 $\rightarrow$ EEEV-102

$\rightarrow$ EEEV-60 \& EEEV-104

$\rightarrow$ EEEV-66 \& EEEV-105

๑ EEEV-68 ఈ EEEV-106

- EEEV-69 — EEEV-107

$\square$ EEEV-73 $* \mathrm{H} 77.99$

Concentration $\left(\mu \mathrm{g} \mathrm{ml}^{-1}\right)$
- EEEV-2

- EEEV-3

A EEEV-4

$\checkmark$ EEEV-5

- EEEV-10

- EEEV-18

- EEEV-19

口 EEEV-21

$\triangle$ EEEV-22 $\nabla \quad$ EEEV-32

EEEV-33

- EEEV-40

- EEEV-43

- EEEV-58

EEEV-60

EEEV-66

EEEV-68

EEEV-69
- EEEV-73

1. EEEV-82

$\triangle$ EEEV-86

$\nabla$ EEEV-102

- EEEV-104

- EEEV-107

$3 \mathrm{~B} 4 \mathrm{C}-4$

$\triangle$ WEEV-23

$\diamond \mathrm{H} 77.39$

Fig. 1 | Characterization of anti-EEEV mAbs. a, Supernatant from anti-EEEV hybridoma cells was screened for binding to a mixture of SINV-EEEV-infected and uninfected BHK-21 cells by flow cytometry. Shown are antibody staining from representative negative (1A2) and positive (13B10, subcloned as EEEV10) hybridomas. Data are representative of two independent experiments. b, Recombinant EEEV E2 (residues 1-338) was refolded and purified by size exclusion chromatography (left panel), and analysed by SDS-polyacrylamide gel electrophoresis under non-reducing (NR) and reducing (R) conditions (right panel). Data are representative of two independent experiments. c, Purified anti-EEEV mAbs were tested for binding to recombinant EEEV E2 glycoprotein by ELISA. Data are the mean and s.d. of two independent experiments performed in duplicate. d, HEK-293T cells were transfected with EEEV, VEEV or WEEV pE2-6K-E1 structural genes and stained with EEEV mAbs, anti-VEEV mAb (3B4C-4), anti-WEEV mAb (WEEV-23) or an isotype control $\mathrm{mAb}$ (anti-HCV, H77.39). Data are from three independent experiments. $M_{\mathrm{w}}$ molecular weight.

tions, we cloned and sequenced the viral RNA. Unexpectedly, all of the sequenced EEEV-18 escape variants (16 of 16 clones) contained a 6-amino acid repeat insertion $\left({ }^{192} \mathrm{GAQVKY}{ }^{197}\right)$ in domain $\mathrm{B}$ (Fig. 4b,c and Supplementary Fig. 5). All EEEV-82 escape variant clones (13 of 13 clones) contained a G192R mutation in E2, whereas the EEEV-102 escape variant contained mutations in both domain A (M68T; 3 of 4 clones) and domain B (L227R; 4 of 4 clones) (Fig. 4b,c and Supplementary Fig. 5). The M68R and G192R mutations were introduced individually into the pE2-6K-E1 plasmid to confirm the loss-of-function phenotype. Mutations in M68R or G192R of the E2 gene resulted in abolished binding of EEEV-18, EEEV-82 and EEEV-102 to cells transfected with the pE2-6K-E1 expression plasmid (Fig. 4d). When the M68T, G192R and L227R mutations were introduced into the SINV-EEEV infectious complementary DNA (cDNA) clone, the resultant viruses showed diminished neutralization by EEEV-18, EEEV-82 and EEEV-102 (Fig. 4e). Finally, we tested whether the four neutralization escape variants were resistant to inhibition by the remaining potently neutralizing $\mathrm{mAbs}$. Although all of the strongly neutralizing domain B mAbs (EEEV-3, EEEV-10, EEEV-22, EEEV-69 and EEEV-86) completely neutralized the escape variants with $\mathrm{EC}_{50}$ values similar to the parental virus, domain A (EEEV-5 and EEEV-66) and domain A/B (EEEV-18, EEEV-58 and EEEV-107) mAbs failed to neutralize the escape variants as efficiently (Supplementary Fig. 6).

MAb protection in mice. We assessed whether the mAbs could confer protection against EEEV infection in vivo (Fig. 5). We tested a subset of $\mathrm{mAbs}$ with differing neutralization potencies using a lethal challenge model in five-week-old CD-1 mice with a highly pathogenic EEEV (strain FL93-939) engineered to express nanoluciferase with little effect on virulence ${ }^{30}$. Mice received a single $100 \mu \mathrm{g}$ $\left(5 \mathrm{mg} \mathrm{kg}^{-1}\right)$ dose of EEEV $\mathrm{mAbs}$ via the intraperitoneal route either before $(-24 \mathrm{~h})$ or after $(+24 \mathrm{~h})$ subcutaneous $\left(10^{3}\right.$ plaque-forming units (PFU) of EEEV) or aerosol (50-100 median lethal dose, $\mathrm{LD}_{50}$ ) inoculation of EEEV. Mice treated with neutralizing anti-EEEV mAbs (EEEV-3, EEEV-22, EEEV-43, EEEV-58, EEEV-73, EEEV-82 and EEEV-86; $\mathrm{EC}_{50}$ values of $2.2-761 \mathrm{ng} \mathrm{ml}^{-1}$ ) before subcutaneous challenge had $80-100 \%$ survival rates, whereas administration of EEEV-26B, a poorly neutralizing $\mathrm{mAb}\left(\mathrm{EC}_{50}>12,500 \mathrm{ng} \mathrm{ml}^{-1}\right)$ showed little protection (Fig. 5a). When mice were subjected to 


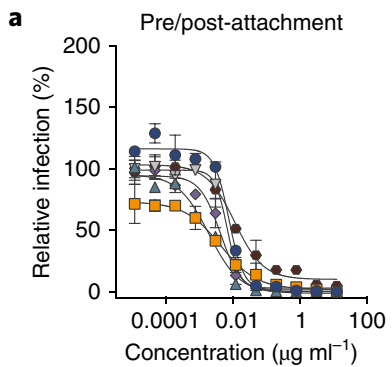

e

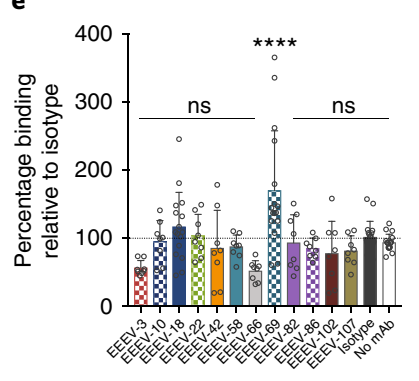

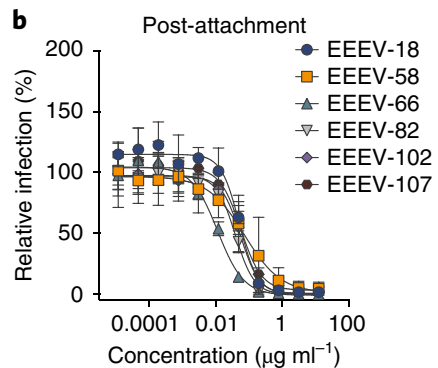

$\mathbf{f}$

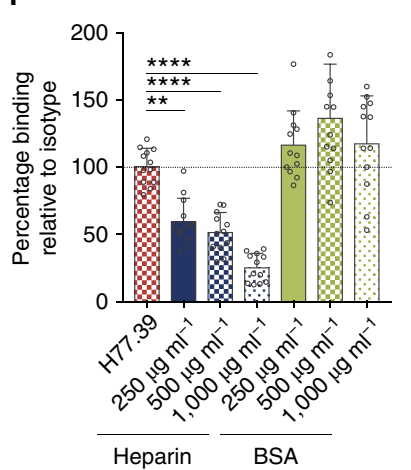

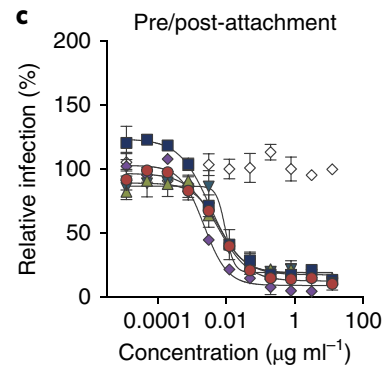

g

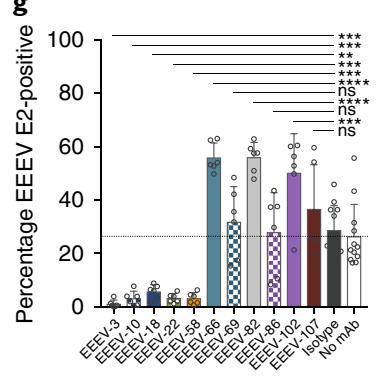

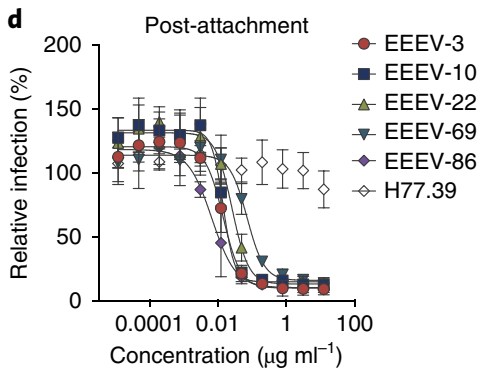

h

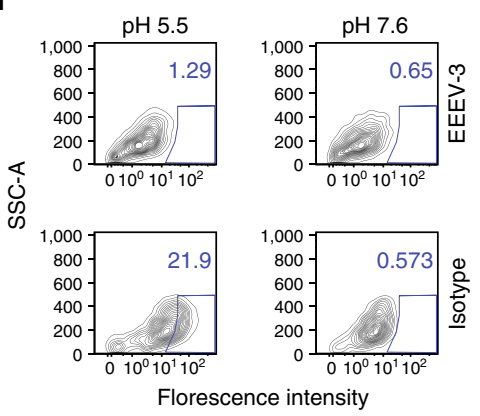

Fig. 2 | Neutralizing activity of anti-EEEV mAbs. MAbs mapping to domain A and A/B (a,b) or domain B (c,d) were evaluated for neutralization. $\mathbf{a}, \mathbf{c}$, Pre/post-attachment neutralization assay. Serial dilutions of anti-EEEV mAbs were incubated with SINV-EEEV and then added to a Vero cell monolayer. Infection was allowed to proceed for $18 \mathrm{~h}$ at which point infected foci were quantitated. Wells were normalized to infected cells containing no mAb. Data are the mean and s.d. of two independent experiments, each performed in duplicate. b,d, Post-attachment neutralization assay. SINV-EEEV was allowed to adsorb onto Vero cells at $4^{\circ} \mathrm{C}$. Unattached virus was removed by washing and diluted anti-EEEV mAbs were added. Infection and processing are as described in panels a and c. Data are the mean and s.d. of two independent experiments, each performed in duplicate. e,f, Attachment inhibition assay.

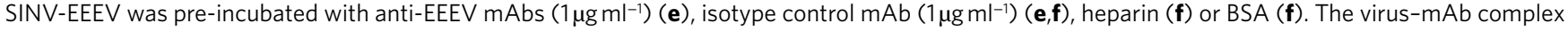
was then added to Vero cells and incubated at $4{ }^{\circ} \mathrm{C}$. Cells were washed and viral RNA was quantitated. Reduction in attachment by anti-EEEV mAbs or heparin was compared to an isotype control mAb (anti-HCV mAb H77.39). Experiments with EEEV-3, EEEV-10, EEEV-22, EEEV-58, EEEV-66, EEEV-82, EEEV-86, EEEV-102 and EEEV-107 are the mean and s.d. of four independent experiments performed in duplicate. Experiments with EEEV-18 and EEEV-69 data are the mean and s.d. of eight independent experiments performed in duplicate. The isotype and no mAb control data are the mean and s.d. of ten independent experiments performed in duplicate (one-way ANOVA with Dunnett's post-test; ${ }^{\star \star} P<0.01$; ${ }^{\star \star \star \star} P<0.0001$ ). NS, not significant. g,h, FFWO. SINV-EEEV was adsorbed to BHK-21 cells for $4{ }^{\circ} \mathrm{C}$. Unbound virus was removed and cells were incubated with anti-EEEV mAbs at $4{ }^{\circ} \mathrm{C}$. FFWO was induced by subjecting the cells to acidic $\mathrm{pH}(\mathrm{pH} 5.5)$ and a $37^{\circ} \mathrm{C}$ degree pulse. As a negative control, cells were subjected to a physiologically relevant $\mathrm{pH}$ ( $\mathrm{pH}$ 7.6). Subsequently, cells were incubated in medium in the presence of $\mathrm{NH}_{4} \mathrm{Cl}$ to prevent subsequent endosomal acidification. Fusion inhibition ( $\mathbf{g}$ ) was determined from flow cytometry data (example with EEEV-3 in h) by staining for EEEV E2-positive cells ( $\mathrm{pH} 5.5$ condition) and subtracting the background at pH 7.6 (average of 3.5\%). Data with anti-EEEV mAbs are the mean and s.d. of three independent experiments performed in duplicate. The isotype and no $\mathrm{mAb}$ control are the mean and s.d. of six independent experiments performed in duplicate. Anti-EEEV mAbs were compared to isotype control (one-way ANOVA with Dunnett's post-test; ${ }^{\star \star} P<0.01 ;{ }^{\star \star \star} P<0.001 ;{ }^{\star \star \star \star} P<0.0001$ ). NS, not significant. SSC-A, side scatter area.

a subcutaneous challenge and administered a single dose of $\mathrm{mAb}$ $24 \mathrm{~h}$ after infection (Fig. 5b) most neutralizing mAbs (EEEV-3, EEEV-18, EEEV-43, EEEV-58, EEEV-73 and EEEV-82) exhibited moderate-to-high levels of protection (40-100\% survival rates), whereas EEEV-22, EEEV-86 and EEEV-26B exhibited less protection. Unexpectedly, the modestly neutralizing EEEV-43 mAb $\left(\mathrm{EC}_{50}\right.$ of $\left.761 \mathrm{ng} \mathrm{ml}^{-1}\right)$ still conferred protection (70\% survival rate) when administered as post-exposure therapy in this model. Additions of $\mathrm{mAb}$ combinations targeting domain A (EEEV-18) and domain B (EEEV-3) and subcutaneous challenge resulted in $100 \%$ protection as prophylaxis and $75 \%$ protection as post-exposure therapy (Fig. 5a,b).

As EEEV is also highly pathogenic via the aerosol route, we examined the efficacy of the mAbs on an aerosol challenge with 50-100 $\mathrm{LD}_{50}$ of EEEV FL93-939. Among the mAbs tested, a majority (EEEV-3, EEEV-5, EEEV-18, EEEV-58 and EEEV-82) protected against death (70-100\% survival) when administered as prophylaxis (Fig. $5 \mathrm{c}$ ). Administration of a mAb combination (EEEV-3 + EEEV-18) as prophylaxis resulted in a $94 \%$ survival rate (Fig. $5 \mathrm{c}$ ). In vivo imaging of mice treated with mAbs EEEV-3, EEEV-18, EEEV-82 and EEEV86 , but not the isotype control $\mathrm{mAb}$, showed marked reductions in viral replication as judged by a decrease in light signal 4 days postinfection (Fig. 5e). However, in the most stringent model of protection, post-exposure therapy at 1 day after aerosol challenge, lower survival rates (10-20\%) were observed with individual neutralizing mAbs EEEV-3, EEEV-5, EEEV-18, EEEV-22, EEEV-58, EEEV-69, EEEV-82 and EEEV-86 or a combination of neutralizing mAbs (EEEV-3+EEEV-18) (Fig. 5d).

\section{Discussion}

EEEV is a highly pathogenic, encephalitic alphavirus that lacks approved vaccines or therapies. We generated a panel of $76 \mathrm{mAbs}$ that bound to EEEV-infected cells, including 18 strongly neutralizing mAbs. Ten of the $18 \mathrm{mAbs}$ exhibited potent neutralizing activity with $\mathrm{EC}_{50}$ values of $<10 \mathrm{ng} \mathrm{ml}^{-1}$. Mapping studies show that these strongly neutralizing $\mathrm{mAbs}$ principally recognized epitopes in domains A and/or B of the E2 glycoprotein. Mechanism of action studies revealed that most of the inhibitory mAbs blocked 
Table 1 | Profiles of strongly neutralizing antibodies against EEEV

\begin{tabular}{|c|c|c|c|c|c|c|}
\hline \multirow[b]{2}{*}{ Antibody } & \multirow[b]{2}{*}{ Isotype $^{a}$} & \multicolumn{2}{|c|}{ Mutagenesis mapping } & \multicolumn{3}{|c|}{ Neutralization against SINV-EEEV } \\
\hline & & E2 domain & $\begin{array}{l}\text { E2 alanine/arginine residues which } \\
\text { reduced } \mathrm{mAb} \text { binding }\end{array}$ & $\mathrm{EC}_{50}\left(\mathrm{ng} \mathrm{ml^{-1 }}\right)$ & $\mathrm{EC}_{90}\left(\mathrm{ng} \mathrm{ml^{-1 }}\right)$ & $\mathrm{EC}_{99}\left(\mathrm{ng} \mathrm{ml} \mathrm{l}^{-1}\right)$ \\
\hline EEEV-3 & $\lg G 2 c$ & B & I180, H181, S182 & 5.6 & 53.2 & 619.5 \\
\hline EEEV-5 & $\lg G 2 c$ & A & K74 & 31.8 & 126.1 & 566.3 \\
\hline EEEV-10 & $\operatorname{lgG} 2 \mathrm{c}$ & $\mathrm{B}$ & I180, H181, S182 & 3.4 & 33.5 & 411.6 \\
\hline EEEV-18 & $\lg G 3$ & $A / B^{b}$ & $\begin{array}{l}\text { M68, G192, A193, Q194, V195, } \\
\text { K196, Y197 }\end{array}$ & 7.7 & 23.2 & 78.1 \\
\hline EEEV-22 & $\lg G 2 c$ & B & I180, H181, S182 & 6.3 & 42.7 & 341.9 \\
\hline EEEV-58 & $\lg G 2 c$ & $A / B$ & $\begin{array}{l}\text { K56, T57, D58, G59, D61, M68, K74, } \\
\text { S75, L81, G192 }\end{array}$ & 4.3 & 66.3 & 1302 \\
\hline EEEV-66 & $\lg G 2 \mathrm{c}$ & A & D58, L81 & 1.9 & 19.6 & 244.8 \\
\hline EEEV-69 & $\lg G 3$ & B & $\mathrm{H} 213, \mathrm{~T} 215$ & 9.3 & 17.7 & 35.7 \\
\hline EEEV-82 & $\lg G 3$ & $A / B$ & M68, L81, G192 & 6.8 & 17.2 & 47.2 \\
\hline EEEV-86 & $\lg G 2 c$ & B & I180, H181, S182 & 2.2 & 12.5 & 82.1 \\
\hline EEEV-102 & $\lg G 3$ & $A / B$ & M68, L81, G192, L227 & 4.3 & 20.3 & 110.7 \\
\hline EEEV-107 & ND & $A / B$ & T57, D58, M68, Q73, S75, L81, G192 & 11.4 & 96.2 & 985.3 \\
\hline
\end{tabular}

aThe $\mathrm{I} g$ isotype was determined by ELISA. ${ }^{\mathrm{b}} \mathrm{A} / \mathrm{B}$ indicates domains $\mathrm{A}$ and $\mathrm{B}$. ND, not done.

infection at a post-attachment stage, with a subset inhibiting viral fusion. Many of the neutralizing mAbs had protective activity against EEEV in vivo, as judged by the outcome in lethal subcutaneous and aerosol challenge models in mice.

Although prior studies have generated mAbs against the EEEV proteins, these mAbs either lacked neutralization activity or were not characterized extensively because of biosafety limitations ${ }^{14-17}$. One cross-reactive, non-neutralizing anti-EEEV mAb that was evaluated had moderate protective efficacy $(\sim 50 \%)$ against VEEV challenge in mice ${ }^{17}$. Presumably, Fc effector functions contributed to the protection against VEEV by this $\mathrm{mAb}$, as has been postulated for non-neutralizing antibodies against other arthritogenic alphaviruses, including Semliki Forest virus ${ }^{31}$ and $\mathrm{CHIKV}^{22}$. Whereas others have immunized mice with recombinant EEEV E2 glycoprotein or inactivated EEEV to obtain $\mathrm{mAbs}^{15,16}$, we speculate that we obtained a large number of neutralizing mAbs because mice were immunized with a replicating virus that displayed EEEV structural proteins in their native form. At present, it remains unclear why we obtained only type-specific neutralizing mAbs.

Neutralizing antibodies against alphaviruses inhibit infection at several stages in the viral replication cycle including attachment, entry, fusion or egress. Our most inhibitory neutralizing mAbs to E2 domains A and/or B did not block viral attachment to cells; instead, they inhibited infection at a post-attachment stage. Plasma membrane fusion assays showed that several of these mAbs block $\mathrm{pH}$-dependent fusion with membranes. Among the mAbs tested that inhibited infection at a post-attachment step, generally, those recognizing epitopes in domain B (EEEV-3, EEEV-10, EEEV-22, EEEV-69 and EEEV-86) showed less potency when antibody was added after the virus attached to the cells. A previous study with domain $\mathrm{B}$ mAbs against CHIKV suggested that bivalent engagement of the virion was necessary for potent neutralization ${ }^{18}$. It is possible that the anti-EEEV $m A b s$ may also require bivalent engagement for complete neutralization; this mode of recognition may be technically difficult to achieve once the virion has attached to cells because some epitopes are unavailable for binding. One of the neutralizing mAbs, EEEV-69, paradoxically increased virus attachment to Vero cells; unexpectedly, increased plasma membrane fusion was observed with EEEV-66, EEEV-82, EEEV-102 and EEEV-107. These results are analogous to prior reports with anti-VEEV and antiSINV mAbs, both of which increased attachment by stabilizing the interaction between the virus and cells ${ }^{32,33}$. The increase in fusion could be due to antibody-induced exposure of cryptic epitopes that facilitates virus binding to the plasma membrane, a mechanism previously reported with a flavivirus ${ }^{34}$. This phenomenon may not impact the neutralizing activity of these mAbs if (1) neutralization occurs at a stage in the entry pathway before fusion or (2) plasma membrane fusion is not equivalent to endosomal fusion.

Some reports have speculated that domains A and B on the E2 glycoprotein contain a site of receptor engagement for multiple alphaviruses ${ }^{11-13}$. A recent study mapped the binding site of Mxra8, a receptor for several arthritogenic alphaviruses, to residues within the A and B domains on CHIKV E2 glycoprotein ${ }^{24}$. Using a combination of alanine-scanning and targeted mutagenesis of E2 and neutralization escape selection, we mapped the epitopes for neutralizing anti-EEEV $m A b s$ to residues within these domains. Regions in the E2 domains A and B have been implicated as epitopes for neutralizing $\mathrm{mAbs}$ against other alphaviruses including VEEV, CHIKV, SINV and Ross River virus ${ }^{18,22,35-37}$. Our most potently neutralizing mAbs (EEEV-5, EEEV-58, EEEV-66, EEEV-82, EEEV-102 and EEEV-107) recognize an epitope in the 'wing region' (residues 51-81) on E2, a solvent-exposed site at the distal tip of the A domain ${ }^{11}$. The neutralizing $\mathrm{mAbs}$ that mapped to domain $\mathrm{B}$ preferentially bound to two epitopes at residues 180-182 (EEEV-3, EEEV-10, EEEV-21, EEEV22 and EEEV-86) or residues 213-215 (EEEV-4, EEEV-19, EEEV21, EEEV-60 and EEEV-69). Cryo-electron microscopy (cryo-EM) studies with two neutralizing anti-VEEV mAbs (F5 and 3B4C-4) showed binding to sites proximal to and within the wing region of domain A (residues 73-120) or to residues 177-223 in domain $\mathrm{B}$, respectively ${ }^{38}$. These $\mathrm{mAbs}$ are thought to neutralize VEEV infection by preventing the structural rearrangements required for fusion.

Through neutralization escape selection, we also mapped neutralizing mAbs (EEEV-18, EEEV-58 and EEEV-102) to residues spanning domains A and B (residues 68, 192-197 and 227). We note that the corresponding M68 residue on the CHIKV p62-E1 structure is located beneath the $\beta$-strand i6 (residues 74-79) and is not solvent-exposed ${ }^{11}$. Residue $\mathrm{M} 68$ is tightly packed against residue L81, a key binding residue for mAbs EEEV-58, EEEV-66, EEEV-82, EEEV-102 and EEEV-107. We hypothesize that the mutation of either residue (M68 or L81) perturbs the conformational display of the domain A 'wing region' epitope. Mutation of the 

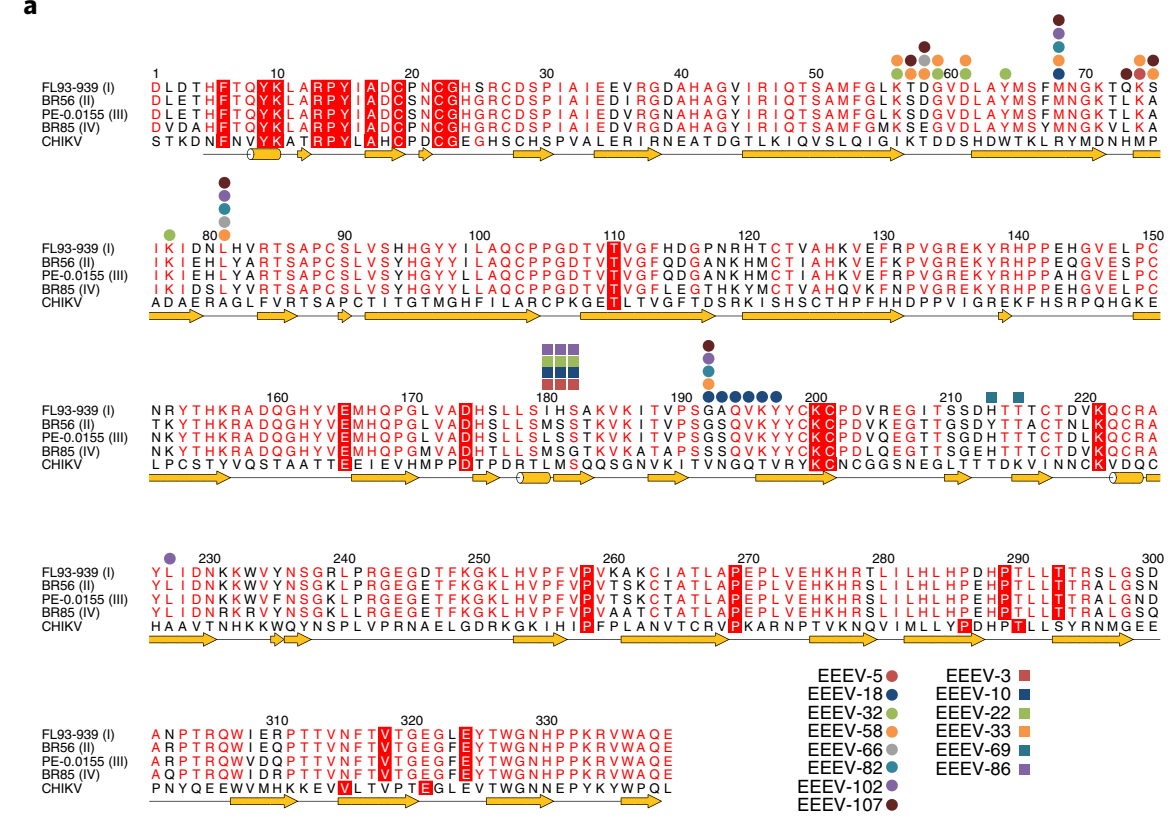

b
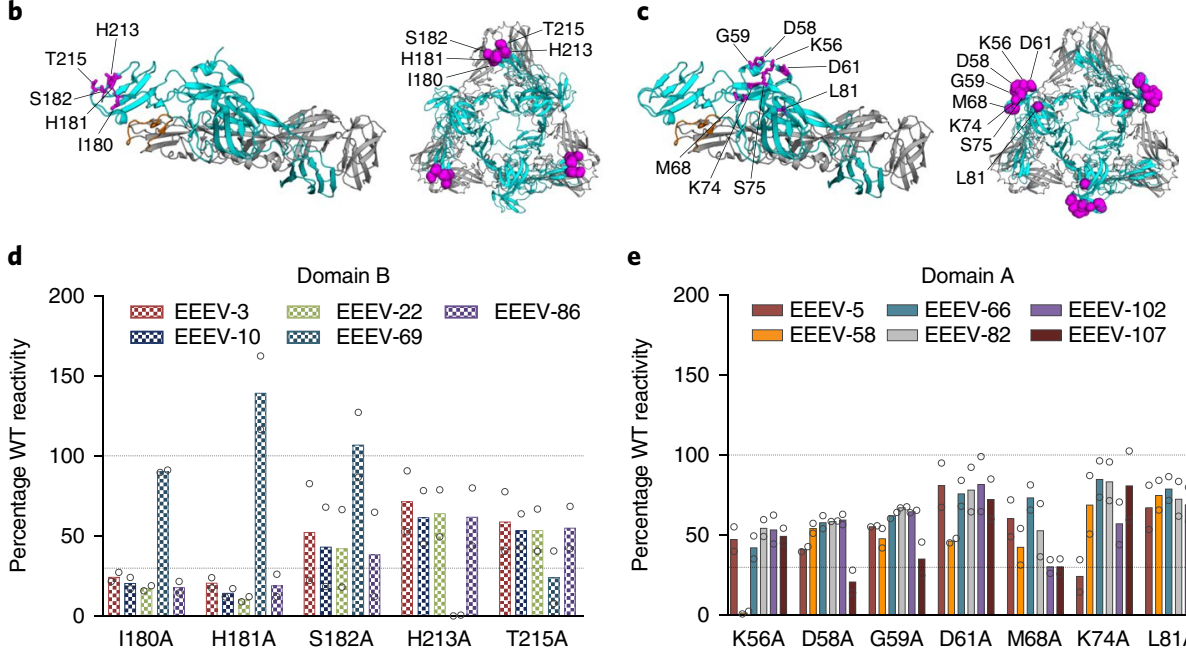

e

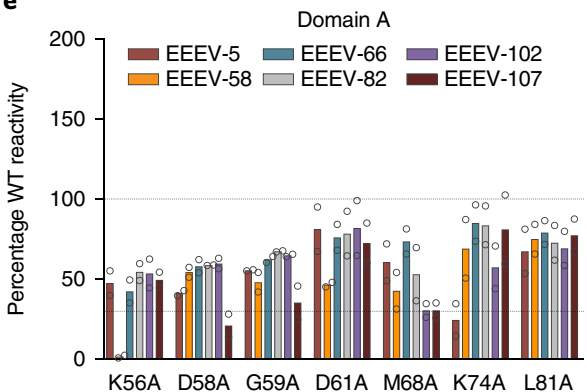

f

g
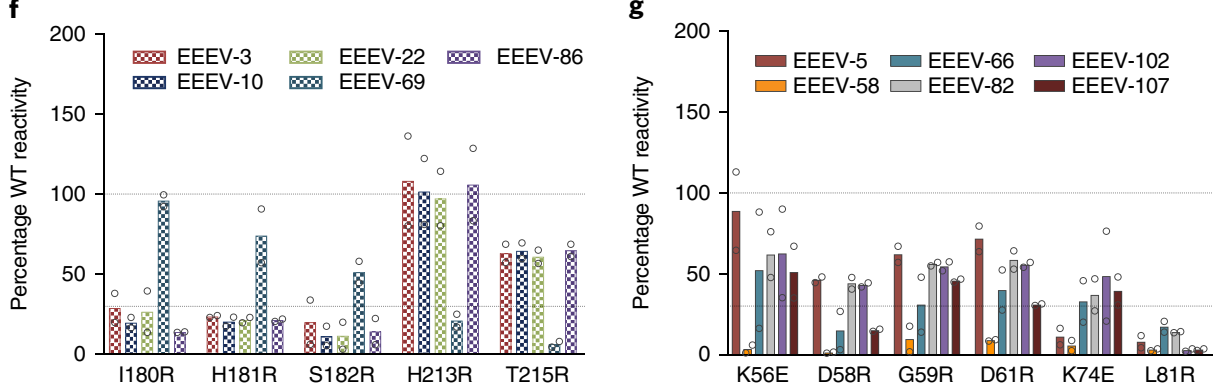

Fig. 3 | Neutralizing mAbs map to domain A or B on the E2 glycoprotein. a, Epitope residues of anti-EEEV mAbs identified by alanine-scanning mutagenesis and viral escape are indicated on the EEEV subtype I (strain FL93-939, GenBank accession no. EF151502), subtype II (strain BR56-BeAn5122, GenBank accession no. AF159559), subtype III (strain PE-0.0155, GenBank accession no. DQ241304) and subtype IV (BR85-436087, GenBank accession no. AF159561) E2 glycoprotein sequences. Anti-EEEV mAbs mapped to domain A or A/B are depicted as circles and mAbs mapped to domain B are depicted as squares. $\mathbf{b}$, Key domain $B$ residues necessary for mAb engagement are highlighted in purple on the CHIKV p62-E1 monomer (PDB 3N41) and trimer (PDB 5ANY). c, Key domain A residues necessary for mAb engagement are also highlighted in purple on the CHIKV p62-E1 monomer (PDB 3N41) and trimer (PDB 5ANY). b,c, The E1 glycoprotein is in grey, the E2 glycoprotein is in cyan and the E1 fusion loop is in orange. $\mathbf{d}$, The binding data of key domain $B$ identified from alanine-scanning mutagenesis are shown for potently neutralizing mAbs. $\mathbf{e}$, The binding data of key domain $A$ residues identified from alanine-scanning mutagenesis are shown for potently neutralizing mAbs. $\mathbf{f}$, The binding data of key domain B identified from arginine or glutamic acid mutagenesis are shown for potently neutralizing $\mathrm{mAbs}$. $\mathbf{g}$, The binding data of key domain A residues identified from arginine or glutamic acid mutagenesis are shown for potently neutralizing mAbs. Residues were identified as critical if $<25 \% \mathrm{mAb}$ binding was observed and $>70 \%$ binding was retained by the oligoclonal EEEV mAb control. Data are the mean and s.d. from two independent experiments. 

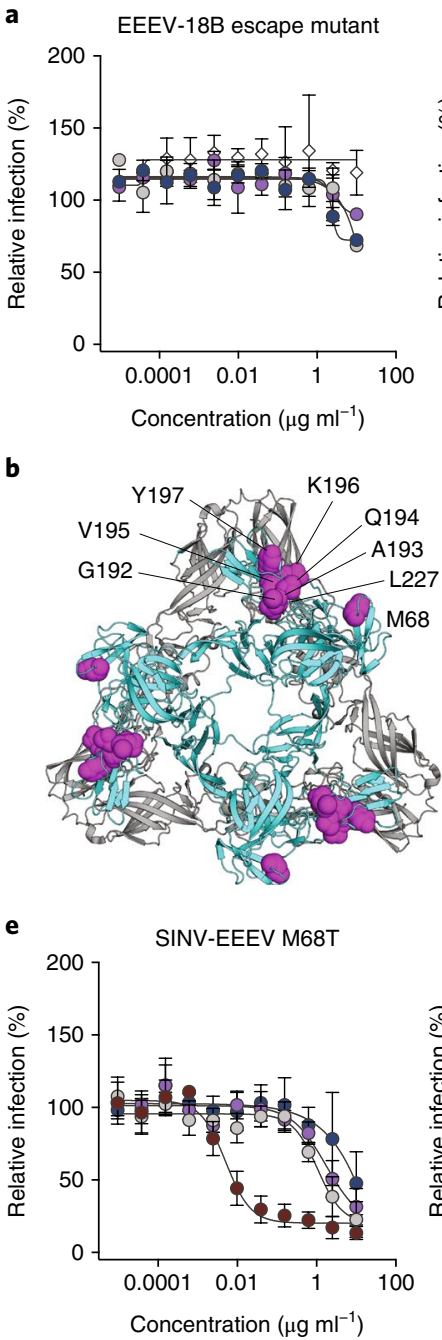
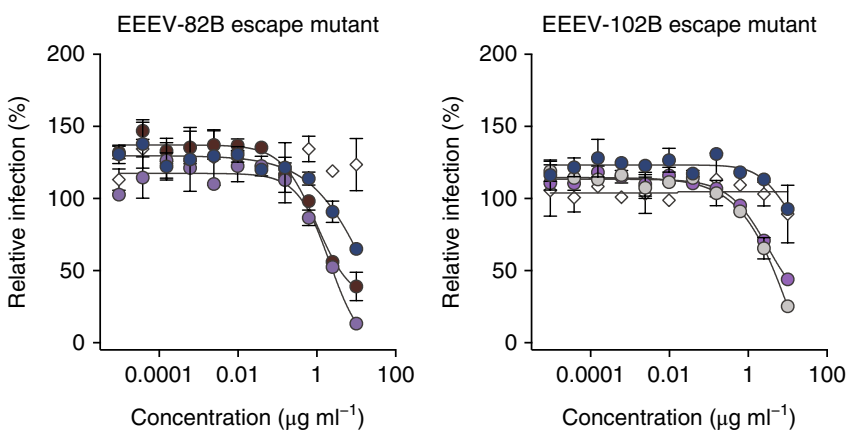

c

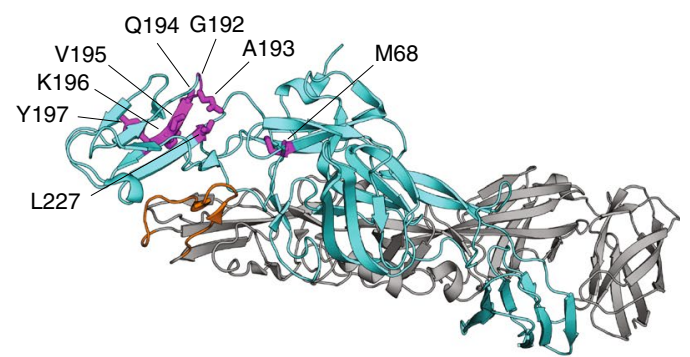

SINV-EEEV G192R

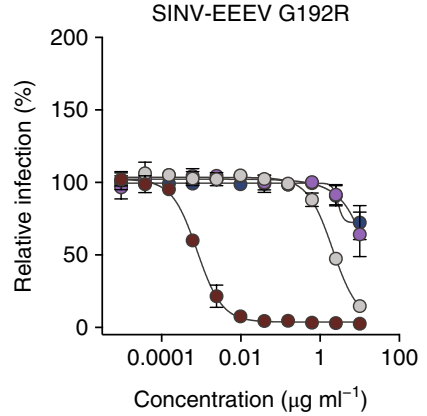

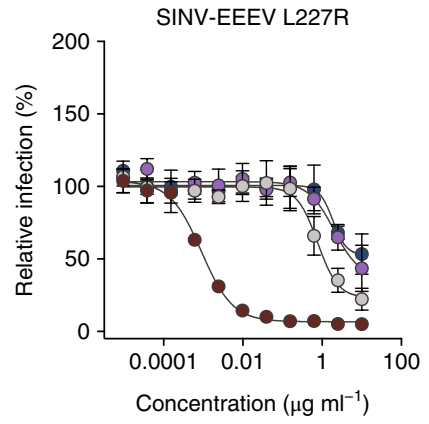

SINV-EEEV WT
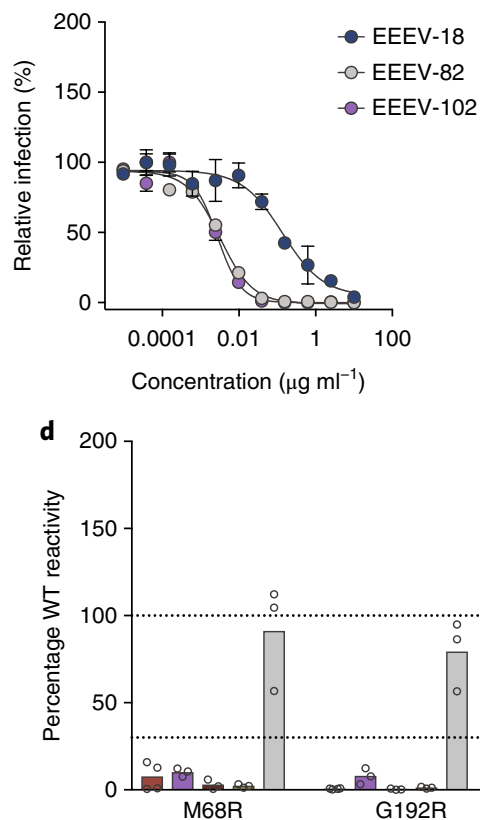

SINV-EEEV WT

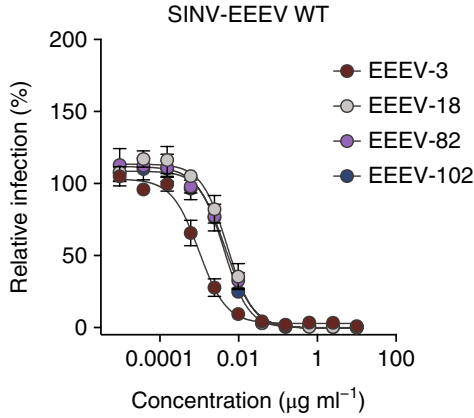

Fig. 4 | Characterization of EEEV mAb escape mutants. a, Neutralization escape virus pools were tested for sensitivity to the mAbs used for selection. Serially diluted $\mathrm{mAbs}$ and $10^{2} \mathrm{FFU}$ of each passaged virus were incubated for $1 \mathrm{~h}$ and then added to Vero cell monolayers. Sixteen hours later, viral antigens containing foci were stained and infection was normalized to infected wells containing no mAb. Data are the mean and s.d. of two independent experiments performed in duplicate. b,c, Neutralization escape mutations were identified by Sanger sequencing. EEEV-18, EEEV-82 and EEEV-102 escape mutations are mapped onto the CHIKV p62-E1 trimer (PDB 5ANY) (b) and the monomer structure (PDB 3N41) (c). The E1 glycoprotein is in grey, the E2 glycoprotein is in cyan and the E1 fusion loop is in orange. d, Neutralization escape mutations were engineered into a structural gene (C-E3-E2-6K-E1) vector and expressed in HEK-293T cells. Cells were stained using the selection mAb and analysed by flow cytometry. Data are the mean and s.d. from three independent experiments, with the exception of EEEV-18 (four experiments). e, Escape mutations were engineered into the SINV-EEEV infectious cDNA clone. Mutant viruses were generated and tested for sensitivity to the mAbs used for selection (EEEV-18, EEEV-82 and EEEV-102) and a domain B mAb (EEEV-3). Data are the mean and s.d. of two independent experiments performed in duplicate.

solvent-exposed residue G192 markedly reduced binding and neutralization of mAbs EEEV-18, EEEV-58 and EEEV-102. In the CHIKV p62-E1 structure, the distance between residues M68 and G192 is $\sim 28 \AA^{11}$. This distance is sufficient for engagement by a fragment antigen-binding (Fab) molecule since the antigen-binding site spans $\sim 35 \AA$.

We also assessed whether the escape variants selected against domain $\mathrm{A} / \mathrm{B}$ mAbs were susceptible to inhibition by the remaining potently neutralizing mAbs. The domain B mAbs (EEEV-3, EEEV-10, EEEV-22, EEEV-69 and EEEV-86) showed no loss in neutralization potency against the escape variants. However, four potently inhibitory mAbs, EEEV-5 (domain A), EEEV-58 (domain A/B), EEEV-66 (domain A) and EEEV-107 (domain A/B), showed reduced ability to neutralize the escape variants. The domain A-specific mAbs EEEV-5 and EEEV-66 did not neutralize the EEEV18 and EEEV-102 escape variants, and the domain A/B-specific
mAbs EEEV-58 and EEEV-107 failed to neutralize all three escape variants. Although we speculate that the binding site of EEEV-66 may be similar to or overlap that of mAbs EEEV-18, EEEV-82 and EEEV-102, higher resolution structural studies (for example, X-ray crystallography or cryo-EM) will be required to determine the precise antibody footprints.

The composite $\mathrm{AB}$ domain epitope, which bridges the two domains, is analogous to the site recognized by the neutralizing anti-CHIKV mAb (CHK-265), which binds and cross-links these domains on adjacent spikes on the virion surface ${ }^{18}$. The crosslinking of two E2 subunits by CHK-265 restricts domain B from undergoing conformational changes and prevents the exposure of the fusion loop located underneath in the E1 subunit. A similar mechanism may occur with the strongly neutralizing EEEV mAbs EEEV-18, EEEV-82, EEEV-102 and EEEV-107. 

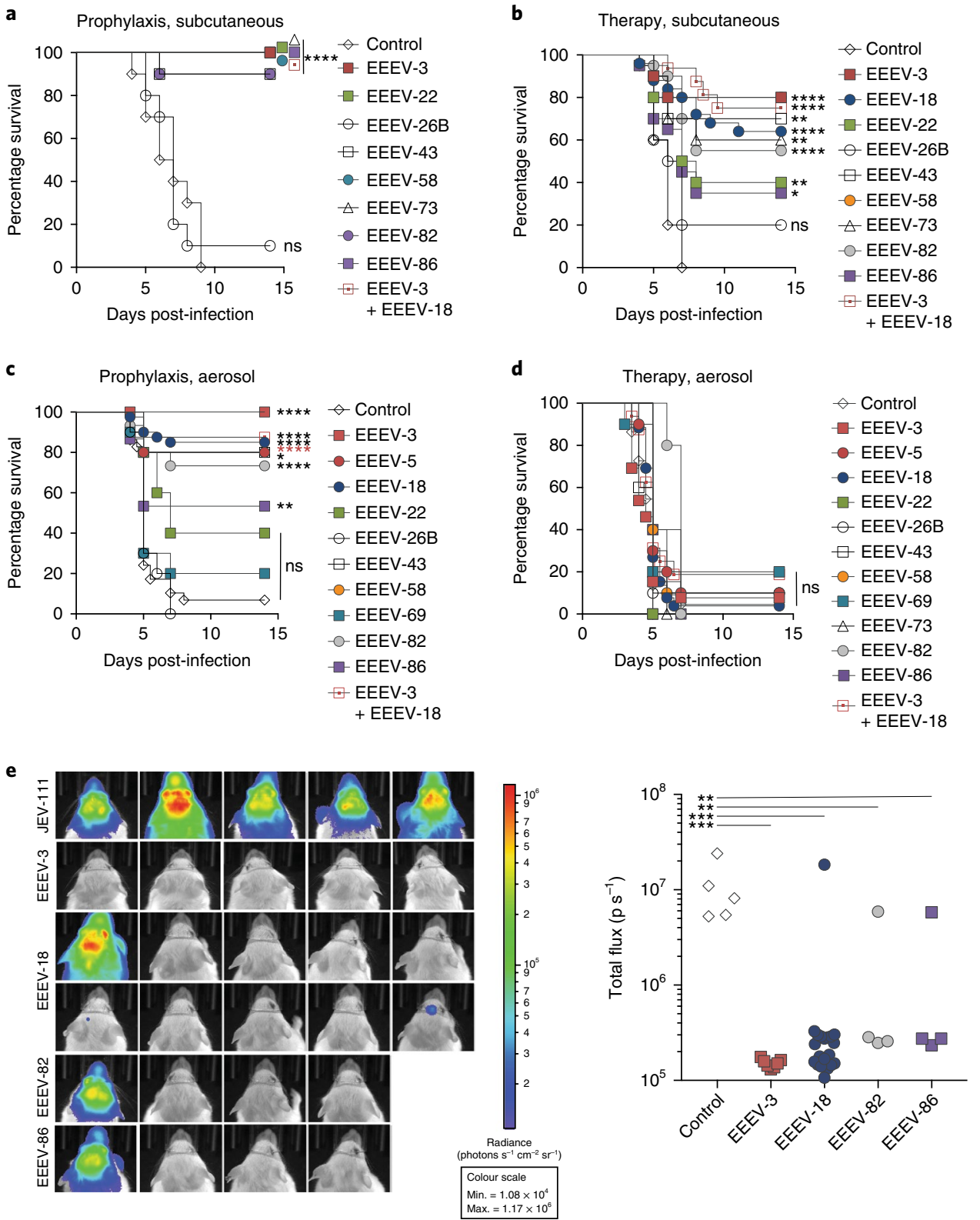

Fig. 5 | Anti-EEEV mAbs exhibit in vivo protection. a-d, Five-week-old female CD-1 mice were administered $100 \mu$ g of the indicated mAbs via an intraperitoneal route either as prophylaxis $(-24 \mathrm{~h}$, left panels) or therapeutically ( $+24 \mathrm{~h}$, right panels) and then challenged with EEEV FL93-939 via subcutaneous $\left(10^{3} \mathrm{FFU}\right)(\mathbf{a}, \mathbf{b})$ or aerosol $\left(50-100 \mathrm{LD}_{50}\right)(\mathbf{c}, \mathbf{d})$ route. Isotype control: $n=10(\mathbf{a}) ; n=20(\mathbf{b}) ; n=29(\mathbf{c}) ; n=22(\mathbf{d})$. EEEV-3: $n=10(\mathbf{a}) ; n=10$ (b); $n=13$ (c); $n=13$ (d). EEEV-5: $n=10$ (c); $n=10$ (d). EEEV-18: $n=25$ (b); $n=40$ (c); $n=26$ (d). EEEV-22: $n=10$ (a); $n=10$ (b); $n=5$ (c); $n=5$ (d). EEEV26B: $n=10$ (a); $n=10$ (b); $n=10$ (c); $n=10$ (d). EEEV-43: $n=10$ (a); $n=10(\mathbf{b}) ; n=5(\mathbf{c}) ; n=5$ (d). EEEV-58: $n=10(\mathbf{a}) ; n=10(\mathbf{b}) ; n=10(\mathbf{c}) ; n=10(\mathbf{d})$. EEEV-69: $n=10$ (c); $n=10$ (d). EEEV-73: $n=10$ (a); $n=10$ (b); $n=5$ (d). EEEV-82: $n=10$ (a); $n=20$ (b); $n=15$ (c); $n=5$ (d). EEEV-86: $n=10$ (a); $n=20$ (b); $n=15$ (c); $n=5$ (d). EEEV-3 + EEEV-18: $n=16$ (a); $n=16$ (b); $n=16$ (c); $n=16$ (d). One-way log rank test with Bonferroni multiple comparison correction: ${ }^{\star} P<0.05$; ${ }^{\star \star} P<0.01$; ${ }^{\star \star \star \star} P<0.0001$. NS, not significant. e, Four or five days post-infection, an IVIS was used to visualize EEEV-luciferase in mice that received prophylactic treatment and were challenged via an aerosol route (left panel). The total flux (photons s ${ }^{-1}$ ) in the head region of each animal was quantified (isotype: $n=5$; EEEV-3: $n=7$; EEEV-18: $n=20$; EEEV-82: $n=4$; EEEV-86: $n=4$ ). One-way ANOVA with Dunnett's post-test: ** $P<0.01$; ${ }^{\star \star \star} P<0.001$.

Several of our highly neutralizing mAbs showed substantial protective efficacy when mice were challenged with EEEV by a subcutaneous or aerosol route. In the lethal subcutaneous challenge models, mAb protection correlated most consistently with potent neutralization activity and binding to residues spanning domains $\mathrm{A}$ and $\mathrm{B}$ of the E2 glycoprotein (EEEV-18, EEEV-58 and EEEV-82). One strongly neutralizing domain $\mathrm{B} \mathrm{mAb}$ (EEEV-3) also protected efficiently in these models. Most of these mAbs (EEEV-3, EEEV-18 and EEEV-58) neutralized infection at a post-attachment stage and efficiently blocked viral plasma membrane fusion. Unexpectedly, EEEV-43, a weakly neutralizing $\mathrm{mAb}\left(\mathrm{EC}_{50}\right.$ of $\left.761 \mathrm{ng} \mathrm{ml}^{-1}\right)$, and EEEV-73 $\left(\mathrm{EC}_{50}\right.$ of $\left.49.7 \mathrm{ng} \mathrm{ml}^{-1}\right)$, a moderately neutralizing $\mathrm{mAb}$, both protected when administered as prophylaxis or therapy. Analogously, a non-neutralizing anti-EEEV mAb protected against subcutaneous EEEV challenge in mice when administered 1 day before infection ${ }^{17}$. Although further studies are warranted, we 
speculate that $\mathrm{Fc}$ effector functions may contribute to the in vivo efficacy of weakly to moderately neutralizing protective mAbs. Alternatively, the neutralization assays with Vero cells may not fully reflect the inhibitory activity against cell targets in vivo.

The post-exposure mAb therapy trials in the context of aerosol challenge of mice showed limited efficacy. After aerosol challenge, encephalitic alphaviruses rapidly enter the brain from the olfactory neuroepithelium via olfactory neurons ${ }^{39,40}$. The treatment failure we observed in the context of aerosol challenge could be due to one of several reasons: (1) the virus spreads rapidly to the brain via olfactory neurons whereas antibody entry is limited by the blood-brain barrier $^{41,42}$; (2) the combination of high levels of virus and limiting amounts of a single $\mathrm{mAb}$ in the brain may result in rapid neutralization escape. Indeed, the use of a single neutralizing anti-CHIKV $\mathrm{mAb}$ promoted escape variants in vivo ${ }^{22,43}$. However, since combination therapy with highly neutralizing domain A- and domain B-reactive antibodies failed to improve clinical outcome after aerosol challenge, virus entry into the brain may represent a point after which $\mathrm{mAb}$ therapy has limited efficacy against EEEV in mice.

Currently, there are no approved vaccines against EEEV. Vaccine efforts against $\mathrm{HIV}$, hepatitis $\mathrm{C}$ virus (HCV) and influenza virus focus on eliciting neutralizing antibodies to protective epitopes on viral envelope proteins through 'reverse vaccinology ${ }^{44-46}$. Our study identifies specific epitopes on the E2 glycoprotein that can be engaged by potently neutralizing EEEV mAbs. Studies are planned to apply this information to the next generation of vaccine design against EEEV and other encephalitic alphaviruses.

\section{Methods}

Animal ethics statement. All animal procedures were carried out in accordance with Association for Assessment and Accreditation of Laboratory Animal Careapproved institutional guidelines for animal care and use and approved by the Institutional Animal Care and Use Committees at the University of Pittsburgh and Washington University School of Medicine. Injections were performed under anaesthesia that was induced and maintained with ketamine hydrochloride and xylazine; all efforts were made to minimize suffering.

Cell lines and plasmids. Vero, HEK-293T and BHK-21 cells were obtained from the American Type Culture Collection and propagated in DMEM supplemented with $5 \%$ (Vero and BHK-21) or 10\% (HEK-293T) foetal bovine serum (FBS; Omega Scientific), $100 \mathrm{U} \mathrm{ml}^{-1}$ penicillin, $100 \mu \mathrm{g} \mathrm{ml}^{-1}$ streptomycin and $10 \mathrm{mM}$ HEPES. All cell lines were tested and judged free of Mycoplasma contamination using a commercial kit. The plasmids pKR780-2-EEEV, pKR780-2-VEEV and pKR780-2-WEEV are comprised of the codon-optimized pE2-6K-E1 genes of EEEV FL93-939, VEEV TrD and WEEV CB87, respectively, under the control of a chicken $\beta$-actin promoter, which have been cloned into the pCAGGS expression vector (Addgene). Replication-competent SINV chimeric viruses were constructed by replacing the SINV TR339 structural protein genes with the EEEV FL93-939 structural protein genes under control of the SINV subgenomic promoter in the TR339 cDNA clone ${ }^{47}$. The cDNA clones of EEEV TrD, FL93-939 wild-type (WT) and nanoluciferase-expressing challenge viruses have been described ${ }^{30,48}$.

Virus production. All viruses were produced by plasmid linearization, in vitro transcription with SP6 or T7 DNA-dependent RNA polymerase and electroporation into BHK-21 cells. Virus mutants were generated using a QuikChange II XL Site-Directed Mutagenesis Kit (Agilent) and verified by DNA sequencing. Virus supernatant (P0) was passaged in Vero cells and collected $24-36 \mathrm{~h}$ after infection. Supernatant was overlaid onto a $20 \%$ sucrose gradient and concentrated at 30,000 r.p.m. for $2 \mathrm{~h}$ using a SW 32 Ti rotor (Beckman Coulter). Viral pellets were resuspended in PBS and stored at $-80^{\circ} \mathrm{C}$. Virus titre was determined by focus-forming or plaque assay.

MAb generation. Six-week-old $\mathrm{Irf3}^{-/-} \mathrm{C} 57 \mathrm{BL} / 6$ female mice were infected and boosted with $10^{5}$ focus-forming units (FFU) of SINV-EEEV and given a final intravenous boost with $10^{6} \mathrm{FFU}$ of SINV-EEEV three days before fusion with myeloma cells. Hybridomas that secreted antibodies reacting with SINV-EEEVinfected BHK-21 cells were identified by flow cytometry and cloned by limiting dilution. MAbs were isotyped by Pierce ELISA (Thermo Fisher Scientific) and hybridomas were sent for commercial preparation and purification by protein $\mathrm{A}$ affinity chromatography (Bio X Cell). All mAbs were screened initially with a single end point neutralization assay using neat hybridoma supernatant $\left(\sim 10 \mu \mathrm{g} \mathrm{ml}^{-1}\right)$, which was incubated with $10^{2} \mathrm{FFU}$ of SINV-EEEV for $1 \mathrm{~h}$ at $37^{\circ} \mathrm{C}$ Virus-mAb complexes were added to Vero cell monolayers in 96-well plates.
After 90 min, cells were overlaid with $1 \%(\mathrm{w} / \mathrm{v})$ methylcellulose in MEM supplemented with $2 \%$ FBS. Plates were collected $18 \mathrm{~h}$ later and fixed with $1 \%$ paraformaldehyde in PBS. The plates were incubated sequentially with murine $\mathrm{mAb}$ EEEV-10 and horseradish peroxidase-conjugated goat anti-mouse IgG in PBS supplemented with $0.1 \%$ saponin and $0.1 \%$ BSA. SINV-EEEV-infected foci were visualized using TrueBlue peroxidase substrate (KPL) and quantitated on an ImmunoSpot 5.0.37 Macroanalyzer (Cellular Technologies). Nonlinear regression analysis was performed after comparison to wells infected with SINV-EEEV in the absence of $\mathrm{mAb}$.

Protein expression and purification. Residues 1-338 encoding the E2 gene of EEEV (strain FL93-939) were cloned into the pET-28a Escherichia coli expression vector and transformed into BL21(DE3) chemically competent cells (Thermo Fisher Scientific). Cells were grown at $37^{\circ} \mathrm{C}$ in lysogeny broth to an $\mathrm{A}_{600}$ of 0.9 and then induced with $1 \mathrm{mM}$ isopropyl- $\beta$-D-thiogalactopyranoside for $4 \mathrm{~h}$. Bacteria were collected, resuspended in $50 \mathrm{mM}$ Tris- $\mathrm{HCl}, 1 \mathrm{mM}$ EDTA, $0.01 \% \mathrm{NaN}_{3}, 1 \mathrm{mM}$ DTT, 25\% sucrose (TENDS) buffer, and lysed in $50 \mathrm{mM}$ Tris- $\mathrm{HCl}, 1 \mathrm{mM}$ EDTA, $0.01 \% \mathrm{NaN}_{3}, 1 \mathrm{mM}$ DTT, $200 \mathrm{mM}$ sodium chloride, $1 \%$ sodium deoxycholate and $1 \%$ Triton $\mathrm{X}-100$. Inclusion bodies were obtained after centrifugation $(6,000 \mathrm{~g}$ for $30 \mathrm{~min}$ ) and then washed in TENDS buffer supplemented with $100 \mathrm{mM} \mathrm{NaCl}$ and $0.5 \%$ Triton X-100. After a final wash in the same buffer without $0.5 \%$ Triton $\mathrm{X}-100, \sim 200 \mathrm{mg}$ of inclusion bodies were denatured in $100 \mathrm{mM}$ Tris- $\mathrm{HCl}, 6 \mathrm{M}$ guanidinium chloride and $20 \mathrm{mM} \beta$-mercaptoethanol for $1 \mathrm{~h}$. Solubilized protein was refolded overnight at $4{ }^{\circ} \mathrm{C}$ into a buffer containing $400 \mathrm{mM} \mathrm{L}$-arginine, $100 \mathrm{mM}$ Tris- $\mathrm{HCl}, 5 \mathrm{mM}$ reduced glutathione, $0.5 \mathrm{mM}$ oxidized glutathione, $10 \mathrm{mM}$ EDTA and $200 \mathrm{mM}$ phenylmethylsulphonyl fluoride. Refolded protein was concentrated using a $10 \mathrm{kDa}$ molecular weight cut-off stirred cell concentrator (EMD Millipore) and purified by HiLoad 16/600 Superdex 75 pg size exclusion chromatography (GE Healthcare).

ELISA. Recombinant E2 glycoprotein $\left(5 \mu \mathrm{g} \mathrm{ml}^{-1}\right)$ was immobilized onto Maxisorp ELISA plates (Thermo Fisher Scientific) overnight in sodium bicarbonate buffer, $\mathrm{pH}$ 9.3. Plates were washed three times with PBS, $0.05 \%$ Tween 20 and blocked with $5 \% \mathrm{BSA} / \mathrm{PBS}$ for $1 \mathrm{~h}$ at $37^{\circ} \mathrm{C}$. Anti-EEEV mAbs were diluted in $2 \% \mathrm{BSA}$ in $\mathrm{PBS}$ and incubated for $1 \mathrm{~h}$ at room temperature. After serial washing, horseradish peroxidase-conjugated goat anti-mouse $\operatorname{IgG}(\mathrm{H}+\mathrm{L} ; 1$ :2,000 dilution; Jackson ImmunoResearch) was added and incubated for $1 \mathrm{~h}$ at room temperature. After washing, plates were developed with Dako 3,3',5,5' - tetramethylbenzidine substrate (Agilent); the reaction was stopped with $2 \mathrm{~N} \mathrm{H}_{2} \mathrm{SO}_{4}$ and absorbance was read at $450 \mathrm{~nm}$ with a TriStar Microplate Reader (Berthold Technologies). For virus capture ELISA, ultracentrifuged SINV-EEEV virions were immobilized directly onto Maxisorp ELISA plates for $1 \mathrm{~h}$ at room temperature. Virus ELISAs were performed similarly, but Tween 20 detergent was omitted from the wash buffer.

Expression of WT or mutant structural proteins. Alanine-scanning mutagenesis was performed on EEEV E2 residues 1-360 with alanine residues mutated into serine. EEEV E2 alanine mutants that exhibited a partial loss-of-binding phenotype (residues $56-62,64,68,73-79,81,192,180-182,212-213$ and 215) were substituted with arginine residues. For residues with positive charges (K56 and K74), a glutamic acid substitution was made. Plasmids containing the codonoptimized EEEV, VEEV or WEEV pE2-6K-E1 structural proteins or EEEV E2 alanine mutants were transfected in HEK-293T cells using Lipofectamine 3000 (Thermo Fisher Scientific). Sixteen hours post-transfection, cells were washed with PBS and fixed with the Foxp3/Transcription Factor Staining Buffer Set (Thermo Fisher Scientific). Cells were washed twice with PBS followed by another wash with permeabilization buffer (Thermo Fisher Scientific). Cells were stained with anti-EEEV mAbs at $1 \mu \mathrm{g} \mathrm{ml}^{-1}$ in permeabilization buffer and incubated for $1 \mathrm{~h}$ at $4{ }^{\circ} \mathrm{C}$. For cross-reactivity studies, anti-VEEV $\mathrm{mAb} 3 \mathrm{~B} 4 \mathrm{C}-4^{20}$ and anti-WEEV mAb (WEEV-23; S.K.A. and M.S.D., unpublished results) were used as positive controls. After two washes with permeabilization buffer, antibodies were detected with Alexa Fluor 647 conjugated goat anti-mouse IgG (1:2,000 dilution; Thermo Fisher Scientific). After two washes, cells were resuspended in $100 \mu \mathrm{l}$ of permeabilization buffer and analysed on a MACSQuant Analyzer (Miltenyi Biotec). Using previously published criteria, alanine mutants with $<25 \%$ reactivity compared to WT that exhibited $>70 \%$ reactivity to a polyclonal anti-EEEV $\mathrm{mAb}$ cocktail were deemed as key binding residues ${ }^{19}$.

Generation of virus escape mutants. To generate neutralization escape mutants, SINV-EEEV $\left(1.2 \times 10^{5} \mathrm{FFU}\right)$ were incubated with $1 \mu \mathrm{g} \mathrm{ml}^{-1}$ of EEEV $\mathrm{mAbs}$ for $1 \mathrm{~h}$ at $37^{\circ} \mathrm{C}$. The virus $-\mathrm{mAb}$ complexes were added to Vero cells. One day post-infection, half of the virus supernatant was incubated with $1 \mu \mathrm{g} \mathrm{ml}^{-1}$ of EEEV mAbs for $1 \mathrm{~h}$ at $37^{\circ} \mathrm{C}$ and added to new Vero cells. The remaining half of the supernatant was frozen at $-80^{\circ} \mathrm{C}$. This process was repeated for 9 days. Escape mutants were confirmed by focus-forming neutralization assays. Viral RNA was isolated from bulk virus supernatant pools using a QIAamp Viral RNA Mini Kit (QIAGEN) and cDNA was generated with an Oligo $(\mathrm{dT})_{20}$ primer using the SuperScript III Reverse Transcriptase kit (Thermo Fisher Scientific). Viral structural genes were amplified using the forward primer 5' -ATGTGCGTCCTGGCCAATATCACGTTTCC-3' and the reverse primer 5'-GAACAAAACTAGGGCAACCACTGCTGTAGC-3'. 
The amplified structural genes were sequenced using four primer sets. Escape mutations were introduced into pKR780-2-EEEV containing the codon-optimized pE2-6K-E1 genes of EEEV FL93-939, expressed in HEK-293T cells, stained with anti-EEEV mAbs and analysed by flow cytometry as described earlier.

Mapping of mutations onto the CHIKV p62-E1 crystal structure. Figures were prepared using the atomic coordinates of the CHIKV p62-E1 monomer (PDB 3N41) and trimer (PDB 5ANY) using the PyMOL software (PyMOL Molecular Graphics System, version 1.7.4; Schrödinger).

Attachment inhibition assays. Vero cells were seeded at $3 \times 10^{5}$ cells per well $24 \mathrm{~h}$ before being assayed. Anti-EEEV mAbs, heparin (Sigma-Aldrich) and BSA (Sigma-Aldrich) were diluted to specified concentrations and incubated for $1 \mathrm{~h}$ at $37^{\circ} \mathrm{C}$ with SINV-EEEV at a multiplicity of infection of 0.01 . The virus-mAb complex was then chilled to $4^{\circ} \mathrm{C}$ and added to pre-chilled Vero cells for $1 \mathrm{~h}$ at $4{ }^{\circ} \mathrm{C}$. After six washes with chilled PBS, RNA was extracted using an RNeasy Mini Kit (QIAGEN). EEEV RNA levels were determined using a TaqMan RNA-to-C $\mathrm{T}_{\mathrm{T}}$ 1-Step Kit (Thermo Fisher Scientific) and an E2-specific primer/probe set ${ }^{26}$. EEEV RNA levels were normalized against glyceraldehyde 3-phosphate dehydrogenase and the relative fold change was compared to cells treated with an isotype control $\mathrm{mAb}$.

Pre/post-attachment and post-attachment neutralization assays. Pre/postattachment neutralization assays were performed by first incubating diluted anti-EEEV mAbs with $10^{2} \mathrm{FFU}$ of SINV-EEEV for $1 \mathrm{~h}$ at $37^{\circ} \mathrm{C}$. The virus-mAb complex was then added to Vero cells for $1.5 \mathrm{~h}$ at $37^{\circ} \mathrm{C}$. Cells were overlaid with $1 \%(\mathrm{w} / \mathrm{v})$ methylcellulose in MEM supplemented with $2 \%$ FBS. Post-attachment neutralization assays were performed by first incubating Vero cells with $10^{2} \mathrm{FFU}$ of SINV-EEEV for $1 \mathrm{~h}$ at $4^{\circ} \mathrm{C}$. Cells were washed extensively with cold DMEM to remove unbound virus. Diluted anti-EEEV mAbs were added to virus-adsorbed cells and incubated for $1 \mathrm{~h}$ at $4{ }^{\circ} \mathrm{C}$. After a $15 \mathrm{~min}$ incubation at $37^{\circ} \mathrm{C}$ to allow virus internalization, cells were overlaid with methylcellulose as previously described. Pre/post-attachment and post-attachment neutralization assays were processed similarly to the single end point neutralization assay described earlier.

Fusion inhibition assays. FFWO assays were performed by first allowing viral adsorption to BHK-21 cells (multiplicity of infection, 25) for $1 \mathrm{~h}$ at $4^{\circ} \mathrm{C}$. Unbound virus was removed by washing with chilled PBS. Diluted mAbs $\left(50 \mu \mathrm{g} \mathrm{ml}^{-1}\right)$ were added to virus-adsorbed cells for $30 \mathrm{~min}$ at $4{ }^{\circ} \mathrm{C}$. Cells were washed with chilled PBS. FFWO was induced by pulsing with fusion medium (Roswell Park Memorial Institute $1640,10 \mathrm{mM}$ HEPES, $0.2 \% \mathrm{BSA}$ and $30 \mathrm{mM}$ succinic acid, $\mathrm{pH}$ 5.5) for $2 \mathrm{~min}$ at $37^{\circ} \mathrm{C}$. A non-fusion control was included using control media (Roswell Park Memorial Institute 1640, $10 \mathrm{mM}$ HEPES, 0.2\% BSA, pH 7.6). After the $37^{\circ} \mathrm{C}$ pulse, cells were washed twice with chilled PBS and incubated in DMEM supplemented with 5\% FBS, $10 \mathrm{mM} \mathrm{HEPES,} 100 \mathrm{U} \mathrm{ml}^{-1}$ penicillin, $100 \mu \mathrm{g} \mathrm{ml}^{-1}$ streptomycin and $20 \mathrm{mM} \mathrm{NH}_{4} \mathrm{Cl}$ to prevent infection via endocytosis. Infection was allowed to proceed for $5 \mathrm{~h}$ and cells were detached and fixed with the Foxp3/ Transcription Factor Staining Buffer Set (Thermo Fisher Scientific). Cells were stained with human mAb EEEV-53 (L.E.W. and J.E.C, unpublished results) at $1 \mu \mathrm{g} \mathrm{ml}^{-1}$ in permeabilization buffer and incubated for $1 \mathrm{~h}$ at $4^{\circ} \mathrm{C}$. After two washes with permeabilization buffer, viral antigen was detected with Alexa Fluor 647 conjugated goat anti-human IgG (1:2,000 dilution; Thermo Fisher Scientific). After two washes with permeabilization buffer, cells were resuspended in $100 \mu$ land analysed on a MACSQuant Analyzer.

Mouse protection studies. Five-week-old female CD-1 mice (Charles River) were administered $100 \mu \mathrm{g}$ of anti-EEEV $\mathrm{mAb}$ or isotype control $\mathrm{mAb}$ via an intraperitoneal route $24 \mathrm{~h}$ pre- or post-challenge. For combined antibody testing, $100 \mu \mathrm{g}$ of each antibody was given as described earlier. Mice were challenged with EEEV FL93-939 WT or a nanoluciferase-expressing version ${ }^{30}$ via a subcutaneous $\left(10^{3} \mathrm{PFU}\right)$ or an aerosol route $\left(50-100 \mathrm{LD}_{50}\right)$. Aerosol exposures were performed as previously described ${ }^{49}$ using the AeroMP exposure system (Biaera Technologies) inside a biological safety cabinet class III. Infected mice were observed at $24 \mathrm{~h}$ intervals through 21 days post-infection; at each time, mice were weighed and mortality was assessed. At 5 days post-challenge, some mice were injected with $10 \mu \mathrm{g}$ Nano-Glo substrate (Promega) subcutaneously and imaged using the in vivo imaging system (IVIS) IVIS SpectrumCT instrument (PerkinElmer) on the auto-exposure setting at $4 \mathrm{~min}$ post-substrate injection. The total flux (photons $\mathrm{s}^{-1}$ ) in the head region, taken as a measure of brain replication, was calculated for animals in each treatment group based on the radiance (photons $\mathrm{sm}^{2} \mathrm{sr}^{-1}$ ) and was quantified using the Living Image Software (PerkinElmer). The dynamic range of the IVIS imager signal from the heads of uninfected mice to highly infected mice was approximately 100 -fold $\left(\sim 1-2 \times 10^{5}\right.$ photons s $^{-1}$ to $\sim 1-2 \times 10^{7}$ photons s $^{-1}$, respectively). Sample sizes were estimated to determine a $50 \%$ reduction in lethality after $\mathrm{mAb}$ treatment. Blinding and randomization were not performed.

Statistical analysis. Statistical significance was determined using Prism version 7.0 (GraphPad Software). Attachment and fusion inhibition assays were analysed using a one-way analysis of variance (ANOVA) test with Dunnett's post-test. In vivo survival experiments were analysed using a one-way log rank test with a
Bonferroni correction. Differences in IVIS signal were analysed using a one-way ANOVA test with Dunnett's post-test.

Reporting Summary. Further information on research design is available in the Nature Research Reporting Summary linked to this article.

\section{Data availability}

The authors declare that all data supporting the findings of this study are available within the paper and its Supplementary Information. The Supplementary Tables provide data on the newly generated $\mathrm{mAbs}$ and mutagenesis (alanine and arginine) mapping of the mAb binding sites on EEEV E2 protein.

Received: 27 May 2018; Accepted: 10 October 2018; Published online: 19 November 2018

\section{References}

1. Garlick, J. et al. Locally acquired eastern equine encephalitis virus disease, Arkansas, USA. Emerg. Infect. Dis. 22, 2216-2217 (2016).

2. Mukerji, S. S., Lam, A. D. \& Wilson, M. R. Eastern equine encephalitis treated with intravenous immunoglobulins. Neurohospitalist 6, 29-31 (2016).

3. Armstrong, P. M. \& Andreadis, T. G. Eastern equine encephalitis virus-old enemy, new threat. N. Engl. J. Med. 368, 1670-1673 (2013).

4. Eastern Equine Encephalitis (Centers for Disease Control and Prevention, 2018); https://www.cdc.gov/easternequineencephalitis/

5. Lindsey, N. P., Staples, J. E. \& Fischer, M. Eastern equine encephalitis virus in the United States, 2003-2016. Am. J. Trop. Med. Hyg. 98, 1472-1477 (2018)

6. Tyler, K. L. Acute viral encephalitis. N. Engl. J. Med. 379, 557-566 (2018).

7. Tan, Y. et al. Large scale complete genome sequencing and phylodynamic analysis of eastern equine encephalitis virus reveal source-sink transmission dynamics in the United States. J. Virol. https://doi.org/10.1128/ JVI.00074-18 (2018).

8. Hunt, A. R., Frederickson, S., Maruyama, T., Roehrig, J. T. \& Blair, C. D. The first human epitope map of the alphaviral E1 and E2 proteins reveals a new E2 epitope with significant virus neutralizing activity. PLoS Negl. Trop. Dis. 4 e739 (2010).

9. Sherman, M. B. \& Weaver, S. C. Structure of the recombinant alphavirus Western equine encephalitis virus revealed by cryoelectron microscopy. J. Virol. 84, 9775-9782 (2010).

10. Zhang, R. et al. $4.4 \AA$ cryo-EM structure of an enveloped alphavirus Venezuelan equine encephalitis virus. EMBO J. 30, 3854-3863 (2011).

11. Voss, J. E. et al. Glycoprotein organization of Chikungunya virus particles revealed by X-ray crystallography. Nature 468, 709-712 (2010).

12. Li, L., Jose, J., Xiang, Y., Kuhn, R. J. \& Rossmann, M. G. Structural changes of envelope proteins during alphavirus fusion. Nature 468, 705-708 (2010).

13. Smith, T. J. et al. Putative receptor binding sites on alphaviruses as visualized by cryoelectron microscopy. Proc. Natl Acad. Sci. USA 92, 10648-10652 (1995)

14. Zhao, J. et al. Phage display identifies an Eastern equine encephalitis virus glycoprotein E2-specific B cell epitope. Vet. Immunol. Immunopathol. 148, 364-368 (2012)

15. EnCheng, S. et al. Analysis of murine B-cell epitopes on Eastern equine encephalitis virus glycoprotein E2. Appl. Microbiol. Biotechnol. 97, 6359-6372 (2013).

16. Roehrig, J. T. et al. Identification of monoclonal antibodies capable of differentiating antigenic varieties of eastern equine encephalitis viruses. Am. J. Trop. Med. Hyg. 42, 394-398 (1990).

17. Pereboev, A. V., Razumov, I. A., Svyatchenko, V. A. \& Loktev, V. B. Glycoproteins E2 of the Venezuelan and eastern equine encephalomyelitis viruses contain multiple cross-reactive epitopes. Arch. Virol. 141, 2191-2205 (1996).

18. Fox, J. M. et al. Broadly neutralizing alphavirus antibodies bind an epitope on E2 and inhibit entry and egress. Cell 163, 1095-1107 (2015).

19. Smith, S. A. et al. Isolation and characterization of broad and ultrapotent human monoclonal antibodies with therapeutic activity against Chikungunya virus. Cell Host Microbe 18, 86-95 (2015).

20. Hunt, A. R., Frederickson, S., Hinkel, C., Bowdish, K. S. \& Roehrig, J. T. A humanized murine monoclonal antibody protects mice either before or after challenge with virulent Venezuelan equine encephalomyelitis virus. J. Gen. Virol. 87, 2467-2476 (2006).

21. Hülseweh, B. et al. Human-like antibodies neutralizing Western equine encephalitis virus. MAbs 6, 718-727 (2014).

22. Pal, P. et al. Development of a highly protective combination monoclonal antibody therapy against Chikungunya virus. PLoS Pathog. 9, e1003312 (2013)

23. Jin, J. et al. Neutralizing monoclonal antibodies block Chikungunya virus entry and release by targeting an epitope critical to viral pathogenesis. Cell Rep. 13, 2553-2564 (2015). 
24. Zhang, R. et al. Mxra8 is a receptor for multiple arthritogenic alphaviruses. Nature 557, 570-574 (2018).

25. Schilte, C. et al. Cutting edge: independent roles for IRF-3 and IRF-7 in hematopoietic and nonhematopoietic cells during host response to Chikungunya infection. J. Immunol. 188, 2967-2971 (2012).

26. Armstrong, P. M., Prince, N. \& Andreadis, T. G. Development of a multi-target TaqMan assay to detect eastern equine encephalitis virus variants in mosquitoes. Vector Borne Zoonotic Dis. 12, 872-876 (2012).

27. Gardner, C. L., Ebel, G. D., Ryman, K. D. \& Klimstra, W. B. Heparan sulfate binding by natural eastern equine encephalitis viruses promotes neurovirulence. Proc. Natl Acad. Sci. USA 108, 16026-16031 (2011).

28. Edwards, J. \& Brown, D. T. Sindbis virus-mediated cell fusion from without is a two-step event. J. Gen. Virol. 67, 377-380 (1986).

29. Fong, R. H. et al. Exposure of epitope residues on the outer face of the chikungunya virus envelope trimer determines antibody neutralizing efficacy. J. Virol. 88, 14364-14379 (2014).

30. Sun, C., Gardner, C. L., Watson, A. M., Ryman, K. D. \& Klimstra, W. B. Stable, high-level expression of reporter proteins from improved alphavirus expression vectors to track replication and dissemination during encephalitic and arthritogenic disease. J. Virol. 88, 2035-2046 (2014).

31. Wust, C. J., Crombie, R. \& Brown, A. Passive protection across subgroups of alphaviruses by hyperimmune non-cross-neutralizing anti-Sindbis serum. Proc. Soc. Exp. Biol. Med. 184, 56-63 (1987).

32. Roehrig, J. T., Hunt, A. R., Kinney, R. M. \& Mathews, J. H. In vitro mechanisms of monoclonal antibody neutralization of alphaviruses. Virology 165, 66-73 (1988)

33. Flynn, D. C., Olmsted, R. A., Mackenzie, J. M.Jr.. \& Johnston, R. E. Antibody-mediated activation of Sindbis virus. Virology 166, 82-90 (1988).

34. Haslwanter, D., Blaas, D., Heinz, F. X. \& Stiasny, K. A novel mechanism of antibody-mediated enhancement of flavivirus infection. PLoS Pathog. 13, e1006643 (2017).

35. Agapov, E. V. et al. Localization of four antigenic sites involved in Venezuelan equine encephalomyelitis virus protection. Arch. Virol. 139, 173-181 (1994).

36. Pence, D. F., Davis, N. L. \& Johnston, R. E. Antigenic and genetic characterization of Sindbis virus monoclonal antibody escape mutants which define a pathogenesis domain on glycoprotein E2. Virology 175, 41-49 (1990).

37. Vrati, S., Fernon, C. A., Dalgarno, L. \& Weir, R. C. Location of a major antigenic site involved in Ross River virus neutralization. Virology 162, 346-353 (1988).

38. Porta, J. et al. Locking and blocking the viral landscape of an alphavirus with neutralizing antibodies. J. Virol. 88, 9616-9623 (2014).

39. Watson, A. M. et al. Ribbon scanning confocal for high-speed high-resolution volume imaging of brain. PLoS ONE 12, e0180486 (2017).

40. Charles, P. C., Walters, E., Margolis, F. \& Johnston, R. E. Mechanism of neuroinvasion of Venezuelan equine encephalitis virus in the mouse. Virology 208, 662-671 (1995).

41. Honnold, S. P. et al. Eastern equine encephalitis virus in mice I: clinical course and outcome are dependent on route of exposure. Virol. J. 12, 152 (2015).

42. Honnold, S. P. et al. Eastern equine encephalitis virus in mice II: pathogenesis is dependent on route of exposure. Virol. J. 12, 154 (2015).
43. Pal, P. et al. Chikungunya viruses that escape monoclonal antibody therapy are clinically attenuated, stable, and not purified in mosquitoes. J. Virol. 88, 8213-8226 (2014).

44. Zhou, T. et al. Structural basis for broad and potent neutralization of HIV-1 by antibody VRC01. Science 329, 811-817 (2010).

45. Law, M. et al. Broadly neutralizing antibodies protect against hepatitis $\mathrm{C}$ virus quasispecies challenge. Nat. Med. 14, 25-27 (2008).

46. Julien, J. P., Lee, P. S. \& Wilson, I. A. Structural insights into key sites of vulnerability on HIV-1 Env and influenza HA. Immunol. Rev. 250, 180-198 (2012).

47. Klimstra, W. B., Ryman, K. D. \& Johnston, R. E. Adaptation of Sindbis virus to BHK cells selects for use of heparan sulfate as an attachment receptor. J. Virol. 72, 7357-7366 (1998).

48. Aguilar, P. V. et al. Structural and nonstructural protein genome regions of eastern equine encephalitis virus are determinants of interferon sensitivity and murine virulence. J. Virol. 82, 4920-4930 (2008).

49. Gardner, C. L. et al. Antibody preparations from human transchromosomic cows exhibit prophylactic and therapeutic efficacy against Venezuelan equine encephalitis virus. J. Virol. 2, e00226-17 (2017).

\section{Acknowledgements}

This work was supported by the Defense Threat Reduction Agency (grant no. HDTRA115-1-0013 to M.S.D. and W.B.K. and grant no. HDTRA1-13-1-0034 to J.E.C) and National Institutes of Health grant no. R01 AI095436 to W.B.K.

\section{Author contributions}

A.S.K., S.K.A., A.Z., M.K.S., D.S.R., D.W.T., W.B.K. and M.S.D. designed the experiments. A.S.K., S.K.A., C.L.G., A.Z., D.W.T., C.S. and K.B. performed the experiments. A.S.K., S.K.A, C.L.G., D.H.F., A.Z., D.W.T. and K.B. performed the data analysis. L.E.W., J.E.C and D.H.F. contributed key reagents. D.S.R. and D.H.F. contributed the methodology. A.S.K. and M.S.D. wrote the initial draft of the manuscript, with the other authors providing comments and edits to the final version.

\section{Competing interests}

M.S.D. is a consultant for InBios International and is on the Scientific Advisory Board of Moderna. J.E.C. has served as a consultant for Takeda Vaccines, Sanofi Pasteur, Pfizer and Novavax, is on the Scientific Advisory Boards of CompuVax, GigaGen, Meissa Vaccines, PaxVax, and is the Founder of IDBiologics.

\section{Additional information}

Supplementary information is available for this paper at https://doi.org/10.1038/ s41564-018-0286-4.

Reprints and permissions information is available at www.nature.com/reprints. Correspondence and requests for materials should be addressed to M.S.D.

Publisher's note: Springer Nature remains neutral with regard to jurisdictional claims in published maps and institutional affiliations.

(c) The Author(s), under exclusive licence to Springer Nature Limited 2018 


\section{Reporting Summary}

Nature Research wishes to improve the reproducibility of the work that we publish. This form provides structure for consistency and transparency in reporting. For further information on Nature Research policies, see Authors \& Referees and the Editorial Policy Checklist.

\section{Statistical parameters}

When statistical analyses are reported, confirm that the following items are present in the relevant location (e.g. figure legend, table legend, main text, or Methods section).
n/a $\mid$ Confirmed
$\square \bigotimes$ The exact sample size $(n)$ for each experimental group/condition, given as a discrete number and unit of measurement
$\square$ \ An indication of whether measurements were taken from distinct samples or whether the same sample was measured repeatedly
$\square$ The statistical test(s) used AND whether they are one- or two-sided
$\square$ Only common tests should be described solely by name; describe more complex techniques in the Methods section.
Х $\square$ A description of all covariates tested
$\square$ \A description of any assumptions or corrections, such as tests of normality and adjustment for multiple comparisons
$\square$ A full description of the statistics including central tendency (e.g. means) or other basic estimates (e.g. regression coefficient) AND
$\square$ variation (e.g. standard deviation) or associated estimates of uncertainty (e.g. confidence intervals)
$\searrow \square$ For null hypothesis testing, the test statistic (e.g. $F, t, r$ ) with confidence intervals, effect sizes, degrees of freedom and $P$ value noted

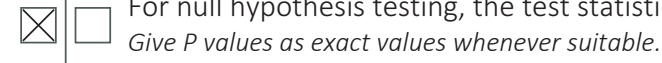
Х $\square$ For Bayesian analysis, information on the choice of priors and Markov chain Monte Carlo settings
\ $\square$ For hierarchical and complex designs, identification of the appropriate level for tests and full reporting of outcomes
$\bigotimes \square$ Estimates of effect sizes (e.g. Cohen's $d$, Pearson's $r$ ), indicating how they were calculated
$\varnothing$ Clearly defined error bars
State explicitly what error bars represent (e.g. SD, SE, CI)

Our web collection on statistics for biologists may be useful.

\section{Software and code}

Policy information about availability of computer code

Data collection GraphPad Prism 7.0

Data analysis PyMOL: v1.7.6.4

For manuscripts utilizing custom algorithms or software that are central to the research but not yet described in published literature, software must be made available to editors/reviewers upon request. We strongly encourage code deposition in a community repository (e.g. GitHub). See the Nature Research guidelines for submitting code \& software for further information.

\section{Data}

Policy information about availability of data

All manuscripts must include a data availability statement. This statement should provide the following information, where applicable:

- Accession codes, unique identifiers, or web links for publicly available datasets

- A list of figures that have associated raw data

- A description of any restrictions on data availability

The authors declare that all data supporting the findings of this study are available within the paper and its Supplementary information. The Supplemental Tables provide data on the newly-generated mAbs, and mutagenesis (alanine and arginine) mapping of the mAb binding sites on EEEV E2 protein. 


\section{Field-specific reporting}

Please select the best fit for your research. If you are not sure, read the appropriate sections before making your selection.

$\bigotimes$ Life sciences $\quad \square$ Behavioural \& social sciences $\quad \square$ Ecological, evolutionary \& environmental sciences

For a reference copy of the document with all sections, see nature.com/authors/policies/ReportingSummary-flat.pdf

\section{Life sciences study design}

All studies must disclose on these points even when the disclosure is negative.

Sample size We used a power calculation (80\% power, 0.05 type I error) to see an 3 to 5 -fold effect in vivo (depending on data distribution), which was an $n=10$.

Data exclusions No data were excluded

Replication All cell culture experiments were repeated multiple independent times. In vivo experiments were performed with independent repeat experiments.

Randomization Not randomized. There was no need to randomized animals for this study. However, the animals were purchased commercially, age- and sexmatched.

Blinding Not blinded. Although the study was not blinded, key experiments were repeated independently by multiple members of the laboratory

\section{Reporting for specific materials, systems and methods}

Materials \& experimental systems

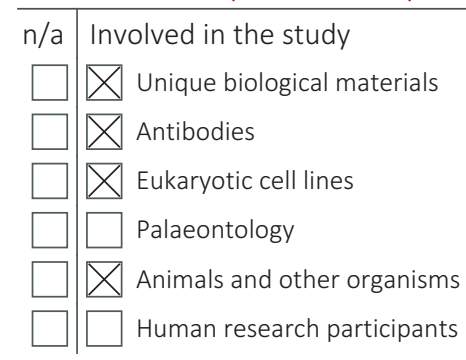

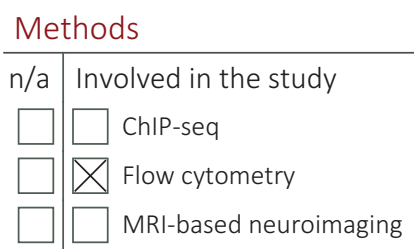

Unique biological materials

Policy information about availability of materials

Obtaining unique materials No restrictions. All materials will be made available through standard MTAs.

\section{Antibodies}

Antibodies used

Anti-EEEV antibodies were validated by Western blotting, binding to recombinant protein, and binding to transfected or infected cells. These EEEV antibodies were generated in this paper

Validation

Describe the validation of each primary antibody for the species and application, noting any validation statements on the manufacturer's website, relevant citations, antibody profiles in online databases, or data provided in the manuscript.

\section{Eukaryotic cell lines}

Policy information about cell lines

Cell line source(s)

Vero cells (ATCC),HEK-293 (ATCC), BHK-21 (ATCC)

Authentication

All cells were purchased from ATCC 
Mycoplasma contamination

Commonly misidentified lines (See ICLAC register)
All cell lines were tested and judged free of mycoplasma contamination using a commercial kit.

Name any commonly misidentified cell lines used in the study and provide a rationale for their use.

\section{Palaeontology}

Specimen provenance

Specimen deposition

Dating methods
Provide provenance information for specimens and describe permits that were obtained for the work (including the name of the issuing authority, the date of issue, and any identifying information).

Indicate where the specimens have been deposited to permit free access by other researchers.

If new dates are provided, describe how they were obtained (e.g. collection, storage, sample pretreatment and measurement), where they were obtained (i.e. lab name), the calibration program and the protocol for quality assurance OR state that no new dates are provided.

Tick this box to confirm that the raw and calibrated dates are available in the paper or in Supplementary Information.

\section{Animals and other organisms}

Policy information about studies involving animals; ARRIVE guidelines recommended for reporting animal research
Laboratory animals
Irf3-/- C57BL/6 mice, CD-1 mice (Charles River Laboratories)

Wild animals

N/A

Field-collected samples

N/A

\section{Human research participants}

Policy information about studies involving human research participants

Population characteristics

N/A

Recruitment

Describe how participants were recruited. Outline any potential self-selection bias or other biases that may be present and how these are likely to impact results.

\section{ChIP-seq}

\section{Data deposition}

$\square$ Confirm that both raw and final processed data have been deposited in a public database such as GEO.

$\square$ Confirm that you have deposited or provided access to graph files (e.g. BED files) for the called peaks.

Data access links

May remain private before publication.

Files in database submission

Genome browser session (e.g. UCSC)

\section{Methodology}

Replicates

Sequencing depth

Antibodies

Peak calling parameters

Data quality

Software
For "Initial submission" or "Revised version" documents, provide reviewer access links. For your "Final submission" document, provide a link to the deposited data.

Provide a list of all files available in the database submission.

Provide a link to an anonymized genome browser session for "Initial submission" and "Revised version" documents only, to enable peer review. Write "no longer applicable" for "Final submission" documents.

Describe the experimental replicates, specifying number, type and replicate agreement.

Describe the sequencing depth for each experiment, providing the total number of reads, uniquely mapped reads, length of reads and whether they were paired-or single-end.

Describe the antibodies used for the ChIP-seq experiments; as applicable, provide supplier name, catalog number, clone name, and lot number.

Specify the command line program and parameters used for read mapping and peak calling, including the ChIP, control and index files used.

Describe the methods used to ensure data quality in full detail, including how many peaks are at FDR 5\% and above 5-fold enrichment.

Describe the software used to collect and analyze the ChIP-seq data. For custom code that has been deposited into a 


\title{
Flow Cytometry
}

Plots

Confirm that:

$\bigotimes$ The axis labels state the marker and fluorochrome used (e.g. CD4-FITC).

Х The axis scales are clearly visible. Include numbers along axes only for bottom left plot of group (a 'group' is an analysis of identical markers).

\All plots are contour plots with outliers or pseudocolor plots.

A numerical value for number of cells or percentage (with statistics) is provided.

Methodology

Sample preparation

\begin{abstract}
Infection was allowed to proceed for $5 \mathrm{~h}$ and cells were detached and fixed with Foxp3/Transcription Factor Staining Buffer Set (Thermo Fisher). Cells were stained with human mAb EEEV-53 (L.E.W. and J.E.C, unpublished results) at $1 \mu \mathrm{g} / \mathrm{ml}$ in permeabilization buffer and incubated for $1 \mathrm{~h}$ at 4 으. After two washes with permeabilization buffer, viral antigen was detected with Alexa Fluor 647 conjugated goat anti-human IgG (1:2000, Thermo Fisher). After two washes with permeabilization buffer, cells were resuspended in $100 \mu$ and analyzed on a MACSQuant Analyzer (Miltenyi Biotec).
\end{abstract}

Instrument

MACSQuant Analyzer (Miltenyi Biotec).

Software

FloJo

Cell population abundance

Describe the abundance of the relevant cell populations within post-sort fractions, providing details on the purity of the samples and how it was determined.

Gating strategy

FSC/SSC, singltes, and live/dead gating

Tick this box to confirm that a figure exemplifying the gating strategy is provided in the Supplementary Information.

\section{Magnetic resonance imaging}

Experimental design

Design type

Design specifications

Behavioral performance measures

Acquisition

Imaging type(s)

Field strength

Sequence \& imaging parameters

Area of acquisition

Diffusion MRI Used

Preprocessing

Preprocessing software

Normalization

Normalization template

Noise and artifact removal
Indicate task or resting state; event-related or block design.

Specify the number of blocks, trials or experimental units per session and/or subject, and specify the length of each trial or block (if trials are blocked) and interval between trials.

State number and/or type of variables recorded (e.g. correct button press, response time) and what statistics were used to establish that the subjects were performing the task as expected (e.g. mean, range, and/or standard deviation across subjects). 
Noise and artifact removal

Volume censoring

Statistical modeling \& inference

Model type and settings

Effect(s) tested

Specify type of analysis:

Statistic type for inference

(See Eklund et al. 2016)

\section{Correction}

Models \& analysis

$\mathrm{n} / \mathrm{a}$ Involved in the study

$\square$ Functional and/or effective connectivity

Х Graph analysis

Х $\square$ Multivariate modeling or predictive analysis (Carlo).
Specify type (mass univariate, multivariate, RSA, predictive, etc.) and describe essential details of the model at the first and second levels (e.g. fixed, random or mixed effects; drift or auto-correlation).

Define precise effect in terms of the task or stimulus conditions instead of psychological concepts and indicate whether ANOVA or factorial designs were used.

Whole brain $\square$ ROI-based $\square$ Both

Specify voxel-wise or cluster-wise and report all relevant parameters for cluster-wise methods.

Describe the type of correction and how it is obtained for multiple comparisons (e.g. FWE, FDR, permutation or Monte 